\title{
Heurísticos y sesgos cognitivos en la dirección de empresas: un meta-análisis
}

\author{
Urra Urbieta, José Anastasio* \\ Medina Lorza, Aida** \\ Acosta Naranjo, Alejandro***
}

\section{Resumen}

En la actualidad, los trabajos sobre el papel que representan los heurísticos y sesgos cognitivos en su relación con la Dirección de Empresas son escasos, fragmentados y parciales. En consecuencia, el objetivo del presente artículo es realizar un meta-análisis sobre los principales hallazgos que marcan el estado de la cuestión y la frontera del conocimiento en dicha relación, haciendo énfasis en los efectos perniciosos de algunos heurísticos sobre el proceso de adopción de decisiones estratégicas, y en la forma de contrarrestar dichos efectos. La metodología empleada, consistente con el objetivo, es el análisis de contenido. Los principales resultados ponen de relieve que, sin duda, el mejor antídoto contra los efectos de los heurísticos y sesgos cognitivos es conocerlos, ser conscientes de cómo actúan y de sus consecuencias. Además, aunque los heurísticos y sesgos cognitivos son inherentemente humanos en su naturaleza, y por tanto difícilmente suprimibles, por su carácter de procesos pueden ser incididos junto a sus efectos (sesgos). Sin embargo, para todo ello, deben ser comprendidos e interiorizados, cuestión no exenta de dificultad dado que resultan conceptualmente abstractos, intangibles, inaprehensibles e inefables. En este sentido, se concluye que las estrategias didácticas interactivas se han demostrado especialmente útiles. Así, mediante una sencilla prueba empírica, además de demostrar como una gran parte de ellos son culturalmente transversales y resistentes al conocimiento.

Palabras clave: Heurísticos, sesgos cognitivos, dirección de empresas, adopción de decisiones estratégicas, procesos.

Recibido: 07-12-10. Aceptado: 30-05-11

* Doctor en Organización de Empresas. Profesor Titular de Escuela Universitaria. Universidad de Valencia - España. E-mail: Anastacio.Urra@uv.es

** Profesora Tiempo Completo. Departamento de Ciencias Económicas y Administrativas. Universidad ICESI (Colombia). E-mail: afmedina@icesi.edu.co

*** Profesor Hora Cátedra. Universidad ICESI (Colombia). Consultor Organizativo.

E-mail: Alejandro.acosta@correo.icesi.edu.co 


\title{
Heuristics and Cognitive Biases in Business Management: A Meta-Analysis
}

\begin{abstract}
Nowadays, studies about the role of heuristics and cognitive biases in relation to business management are scarce, fragmented and partial. Consequently, the objective of this article is to perform a meta-analysis about the main findings that establish the state of the matter and the frontier of knowledge in such a relationship, emphasizing the pernicious effects of some heuristics on the process of adopting strategic decisions, and methods for counteracting these effects. The methodology, consistent with the objective, is content analysis. Principle results show clearly that the best antidote for the pernicious effects of heuristics and cognitive biases is to know them, be aware of how they work and of their consequences. Furthermore, although heuristics and cognitive biases are inherently human in nature and therefore, difficult to elude, due to their process character, they, as well as their effects (biases), can be influenced by management. To do this, however, they must be understood and interiorized - not an easy task, considering that they are conceptually abstract, intangible, hard to grasp and ineffable. Through a simple empirical proof, the study demonstrates that heuristics and cognitive biases are culturally transversal and knowledge resistant, and concludes by offering some interesting didactic clues for explaining and describing them.
\end{abstract}

Key words: Heuristics, cognitive biases, business management, strategic decision adoption, processes.

\section{Introducción}

Desde la filosofía, en Platón (Siglo IV a. de C.), se puede distinguir en su estudio una diferenciación entre lo racional (actividad consiente intelectiva) y lo irracional (representado en lo sensitivo), ambos como procesos clave de carácter cognitivo para la interpretación del mundo en el que nos encontramos inmersos los individuos.

En este sentido, y refiriéndose a Platón, Walter Lippmann (1922), en su obra Opinión Publica, Cap. XXVIII, "El Ilamado a la razón", pone de manifiesto las limitaciones de la racionalidad para abordar lo irracional, y la participación de la intuición en la adopción de decisiones.

De manera contraria, la teoría económica clásica ortodoxa (1870-1920), da paso a la Teoría de la Utilidad Subjetiva Esperada, al asumir que los individuos somos capaces de comprender totalmente el entorno en el que nos desenvolvemos, y de calcular de manera sorprendente las diferentes opciones para encontrar el mejor curso de acción, desconociendo lo que hasta entonces se denominó irracional y su participación en la adopción de decisiones.

Años más tarde, el estudio de los procesos de decisión directiva, desde los planteamientos de la teoría del comportamiento organizativo liderada por la "Escuela de Carnegie", cuestionó esa visión económica ortodoxa y demostró que las decisiones directivas complejas también dependen, además de factores económicos, de factores de conducta (Simon, 1957, 1983; Cyert \& March, 1963). En 
Heurísticos y sesgos cognitivos en la dirección de empresas: un meta-análisis Urra Urbieta, José Anastasio; Medina Lorza, Aida y Acosta Naranjo, Alejandro

este sentido, Simon $(1957,1983)$ indica que las personas somos parcialmente racionales, actuando en muchos de los casos por impulsos emocionales, ya que la racionalidad se ve limitada por la disponibilidad de la información, por la capacidad cognoscitiva y por la disponibilidad de tiempo para tomar las decisiones. Consecuentemente, Simon $(1957,1983)$ propone el Modelo del Comportamiento Satisfactor, antecedente ya del concepto de racionalidad de proceso, tomando como punto de partida los supuestos, más preceptos, de la Teoría de la Utilidad Subjetiva Esperada, y sometiéndolos a crítica profunda, realista y analítica.

Posteriormente, Simon (1978) formaliza esa forma de racionalidad, la racionalidad de proceso, menos restrictiva que la subyacente en la Teoría de la Utilidad Subjetiva Esperada y más acorde con la concepción de racionalidad limitada. Aportación, ésta, valiosa para la comprensión sobre los procesos de adopción de decisiones en las organizaciones, y la fijación de bases para el posterior tratamiento de los procesos de simplificación cognitiva (Schwenk, 1984, 1988). Sin embargo, dichos aportes, adolecen aún de referencias teóricas o empíricas que permitan una aproximación comprensiva al funcionamiento cognitivo del juicio humano y la adopción de decisiones bajo incertidumbre.

A través de los años, el concepto de heurísticos ha venido reflejando cambios desde su concepción ontológica hasta su estructuración lingüística como significante y significado en su relación con la racionalidad. Desde la preocupación por el concepto de la filosofía hasta su adopción por la dirección de empre- sas, pasando por su desarrollo en el campo de la Psicología. En la dirección de empresas se reconoce la misma naturaleza propiamente humana de los heurísticos y sesgos cognitivos, y su papel normativo y simplificador, de selección, procesamiento y ajuste de la información, sobre la percepción y el juicio humano en situaciones de riesgo e incertidumbre. $A$ partir de estas premisas de partida, la preocupación ha devenido en intentar comprender los parámetros organizativos y de gestión que pueden influir sobre el desarrollo de ciertos heurísticos cognitivos, ya que, como demostramos con una pequeña prueba empírica, una gran parte de ellos son culturalmente transversales.

Desde la dirección de empresas se ha adoptado el testigo obligatorio en el terreno de los heurísticos cognitivos, para encontrarnos con una actualidad enfocada en contribuir a su comprensión y gestión, ya que, conocerlos, ser conscientes de cómo actúan, de sus consecuencias, y gestionar los factores de contingencia, configurables y por tanto controlables por la dirección, bajo cuya influencia parecen encontrarse, es el mejor antídoto contra sus efectos perniciosos en los procesos organizativos de adopción de decisiones. Para ello se recurre a conocimientos de la psicología, de la psicología social y de la sociología, y se requiere que dichos heurísticos y sus efectos, sean enseñados y explicados mediante una estrategia didáctica interactiva, basada en experimentos y simulaciones de adopción de decisiones sencillas.

Sin embargo, los factores profundos que rigen estos procesos, sus verdaderas causas y patrones, siguen sin reve- 
lar. Quizás en espera de mayores desarrollos en los campos de la neurología, la genética u otras ciencias que agreguen valor al actual cuerpo de conocimiento.

\section{Heurísticos y sesgos cognitivos}

En este sentido, el análisis de la cognición, que tiene sus orígenes en la psicología, se ha comenzado a explorar en el campo de la estrategia y de la dirección de empresas, teniendo como una de sus principales corrientes de investigación la relativa a los procesos cognitivos, que se refieren a la manera en que el conocimiento es adquirido y utilizado (Schneider \& Angelmar, 1993), y que desde la Behavioural Decision Theory ha supuesto el origen de la línea de trabajo que se ha ocupado del estudio de los efectos de los heurísticos y sesgos cognitivos sobre la estrategia y sobre la adopción de decisiones, tratando de catalogar la miríada de formas en que los individuos violamos sistemáticamente los axiomas de la Teoría de la Utilidad Subjetiva Esperada, como la consistencia y transitividad de las preferencias, y/o sus predicciones (Kahneman \& Lovallo, 1993). A continuación, en el Cuadro 1, se presenta una síntesis de los principales heurísticos y sesgos cognitivos asociados a ellos y de sus efectos sobre la adopción de decisiones.

Entre los trabajos que han configurado las bases teóricas de esta línea de estudio en el seno de la psicología, cabe destacar los de Tversky \& Kahneman (1974), Langer (1975, 1994), y Nisbett \& Ross (1980), entre otros.

Tversky \& Kahneman (1974) sugieren que los heurísticos cognitivos son ciertas normas simplificadoras de selección y procesamiento de la información que, en situaciones de riesgo e incertidumbre, conducen a determinados sesgos de valoración y predicción. Señalan, adicionalmente, que, aunque los heurísticos pueden proporcionar atajos eficientes en el procesamiento de información, en ocasiones conducen a errores severos y sistemáticos. Además, definen, describen, explican, y ofrecen ejemplos de los heurísticos de representatividad, de disponibilidad, y de anclaje y ajuste, y de los múltiples sesgos cognitivos asociados a dichos heurísticos. En su desarroIlo, se remiten recurrentemente a la evidencia derivada de experimentos de laboratorio, y concluyen que el origen de los sesgos cognitivos con efectos perniciosos sobre los procesos de adopción de decisiones individuales se encuentra en el uso de esos heurísticos combinado con deficiencias de la intuición. Cabe señalar cómo algunos investigadores (Gigerenzer, 1991, 1993, 1994) han tratado de cuestionar y desvirtuar tales hallazgos, obteniendo, de manera contraria a su propósito, un rechazo contundente, demostrado, fundamentado y público a sus planteamientos, tal como se establece en la crítica al primero por Tversky \& Kahneman (1996).

Por su parte, Langer (1975, 1994) establece, igualmente mediante experimentos de laboratorio, que, enfrentados a distintas tareas de decisión, los sujetos tendemos a sobrestimar la influencia o impacto de nuestras capacidades y habilidades sobre los resultados de sucesos puramente aleatorios, debido a dos razones: 1) las personas mantenemos una fuerte motivación por controlar el entorno, para 
Heurísticos y sesgos cognitivos en la dirección de empresas: un meta-análisis

Urra Urbieta, José Anastasio; Medina Lorza, Aida y Acosta Naranjo, Alejandro

\begin{tabular}{|c|c|c|}
\hline \multicolumn{3}{|c|}{$\begin{array}{c}\text { Cuadro } 1 \\
\text { Principales heurísticos y sesgos cognitivos y sus efectos } \\
\text { sobre la adopción de decisiones }\end{array}$} \\
\hline $\begin{array}{l}\text { Heurísticos y sesgos } \\
\text { cognitivos }\end{array}$ & Descripción & Efecto \\
\hline $\begin{array}{l}\text { Heurístico de } \\
\text { representatividad }\end{array}$ & $\begin{array}{l}\text { Los juicios de probabilidad sobre un su- } \\
\text { ceso se ven afectados por el grado en } \\
\text { que un caso particular es representativo } \\
\text { de una clase. }\end{array}$ & $\begin{array}{l}\text { El uso de este heurístico puede generar } \\
\text { una alta propensión a desarrollar am- } \\
\text { plias, y en ocasiones muy detalladas, } \\
\text { generalizaciones sobre una persona o } \\
\text { fenómeno con base en sólo unos pocos } \\
\text { atributos de tal persona o fenómeno. }\end{array}$ \\
\hline $\begin{array}{l}\text { Insensibilidad a la } \\
\text { probabilidad previa } \\
\text { de los resultados }\end{array}$ & $\begin{array}{l}\text { Pasar por alto la probabilidad previa de } \\
\text { los resultados (base-rate frequency), } \\
\text { que no tiene efecto sobre la representati- } \\
\text { vidad y sí lo tiene sobre la probabilidad } \\
\text { actual. }\end{array}$ & $\begin{array}{l}\text { Conduce al error de obviar la distribución } \\
\text { base de la población en la estimación de } \\
\text { probabilidad de un caso particular. }\end{array}$ \\
\hline $\begin{array}{l}\text { Ley de los "pequeños" } \\
\text { números }\end{array}$ & $\begin{array}{l}\text { Sobrestimación del grado en que peque- } \\
\text { ñas muestras son representativas de la } \\
\text { población. }\end{array}$ & $\begin{array}{l}\text { Conduce al error de extraer conclusio- } \\
\text { nes firmes a partir de un número reduci- } \\
\text { do de inputs de información. }\end{array}$ \\
\hline Efectos del azar & $\begin{array}{l}\text { Esperar que una corta secuencia de su- } \\
\text { cesos aleatorios sea representativa de } \\
\text { las características esenciales del proce- } \\
\text { so global. }\end{array}$ & $\begin{array}{l}\text { Conduce al error común de contemplar } \\
\text { el azar como un proceso autocorrector } \\
\text { en el que una desviación en una direc- } \\
\text { ción induce una desviación en la direc- } \\
\text { ción opuesta para restaurar el equilibrio. } \\
\text { Sin embargo, en el desarrollo de un pro- } \\
\text { ceso aleatorio las desviaciones no son } \\
\text { corregidas, son meramente diluidas. }\end{array}$ \\
\hline Efectos de regresión & $\begin{array}{l}\text { Pasar por alto el fenómeno de regresión } \\
\text { a la media: las desviaciones positivas } \\
\text { persistentes pueden deberse a razones } \\
\text { aleatorias que, de ser así, aumentan la } \\
\text { probabilidad de una desviación negati- } \\
\text { va; de forma similar, las desviaciones } \\
\text { negativas podrían aumentar la probabili- } \\
\text { dad de una desviación positiva. }\end{array}$ & $\begin{array}{l}\text { Conduce a la invención de explicaciones } \\
\text { causales espurias. }\end{array}$ \\
\hline $\begin{array}{l}\text { Heurístico de } \\
\text { disponibilidad }\end{array}$ & $\begin{array}{l}\text { Los juicios de probabilidad sobre un su- } \\
\text { ceso se ven afectados por la información } \\
\text { disponible. }\end{array}$ & $\begin{array}{l}\text { El uso de este heurístico puede generar } \\
\text { juicios basados en información fácilmen- } \\
\text { te disponible en la memoria o a través de } \\
\text { fuentes externas y coartar una búsque- } \\
\text { da y recolección razonables de informa- } \\
\text { ción. }\end{array}$ \\
\hline Sesgo de proximidad & $\begin{array}{l}\text { Los acontecimientos más próximos, } \\
\text { temporal, espacial y/o afectivamente, } \\
\text { predominan sobre los más distantes, } \\
\text { que son minimizados e ignorados. }\end{array}$ & $\begin{array}{l}\text { Conduce a una fácil y pronunciada dis- } \\
\text { torsión de la probabilidad asignada a un } \\
\text { suceso. }\end{array}$ \\
\hline Sesgo de confirmación & $\begin{array}{l}\text { Tendencia a buscar la información que } \\
\text { confirma las creencias y puntos de vista } \\
\text { previos. }\end{array}$ & $\begin{array}{l}\text { Conduce a limitar la búsqueda de infor- } \\
\text { mación y a descartar la información que } \\
\text { apunta contra las creencias establecidas. }\end{array}$ \\
\hline
\end{tabular}




\begin{tabular}{|c|c|c|}
\hline \multicolumn{3}{|c|}{$\begin{array}{c}\text { Cuadro } 1 \text { (Continuación) } \\
\text { Principales heurísticos y sesgos cognitivos y sus efectos } \\
\text { sobre la adopción de decisiones }\end{array}$} \\
\hline $\begin{array}{c}\text { Heurísticos y sesgos } \\
\text { cognitivos }\end{array}$ & Descripción & Efecto \\
\hline $\begin{array}{l}\text { Correlaciones } \\
\text { ilusorias }\end{array}$ & $\begin{array}{l}\text { La facilidad con que se desarrollan las } \\
\text { operaciones mentales de recuperación y } \\
\text { asociación de información pueden llevar } \\
\text { a creer que sucesos no relacionados es- } \\
\text { tán correlacionados. }\end{array}$ & $\begin{array}{l}\text { Conduce a establecer relaciones causa- } \\
\text { les entre sucesos que en realidad no tie- } \\
\text { nen relación alguna. }\end{array}$ \\
\hline $\begin{array}{l}\text { Heurístico de anclaje } \\
\text { y ajuste }\end{array}$ & $\begin{array}{l}\text { En numerosas situaciones las estima- } \\
\text { ciones se realizan comenzando por un } \\
\text { valor inicial que se ajusta hasta alcanzar } \\
\text { la estimación final. }\end{array}$ & $\begin{array}{l}\text { El uso de este heurístico genera que, de- } \\
\text { pendiendo del punto de partida, se al- } \\
\text { cancen diferentes estimaciones sobre } \\
\text { determinado suceso. }\end{array}$ \\
\hline $\begin{array}{l}\text { Sesgo de anclaje } \\
\text { o de conservadurismo }\end{array}$ & $\begin{array}{l}\text { Las estimaciones o pronósticos se "an- } \\
\text { clan" en la información inicial, a la que se } \\
\text { le atribuye un valor excesivo en el proce- } \\
\text { so de diagnóstico. }\end{array}$ & $\begin{array}{l}\text { Conduce a no revisar las estimaciones o } \\
\text { pronósticos a la luz de la nueva informa- } \\
\text { ción. }\end{array}$ \\
\hline $\begin{array}{l}\text { Sesgos en la } \\
\text { evaluación de } \\
\text { sucesos conjuntos } \\
\text { y disjuntos }\end{array}$ & $\begin{array}{l}\text { Error en estimación de probabilidades. } \\
\text { La probabilidad de un suceso conforma- } \\
\text { do por sucesos conjuntos es siempre in- } \\
\text { ferior a la probabilidad de cada uno de } \\
\text { los sucesos elementales que lo forman; } \\
\text { la probabilidad de un suceso conforma- } \\
\text { do por sucesos disjuntos es siempre su- } \\
\text { perior a la probabilidad de cada uno de } \\
\text { los sucesos elementales que lo forman. }\end{array}$ & $\begin{array}{l}\text { Como consecuencia del anclaje en la } \\
\text { probabilidad de los sucesos elementa- } \\
\text { les, se tiende a sobrestimar la probabili- } \\
\text { dad en los problemas conformados por } \\
\text { sucesos conjuntos y a subestimar la pro- } \\
\text { babilidad en problemas conformados } \\
\text { por sucesos disjuntos. }\end{array}$ \\
\hline $\begin{array}{l}\text { Exceso de } \\
\text { confianza }\end{array}$ & $\begin{array}{l}\text { No se trata de un heurístico sino de un } \\
\text { proceso por el cual una familia de ses- } \\
\text { gos cognitivos genera una confianza fal- } \\
\text { sa o ilusoria en el propio juicio. }\end{array}$ & $\begin{array}{l}\text { Los sesgos que conducen al exceso de } \\
\text { confianza generan la sobrestimación de } \\
\text { las habilidades propias, del conocimien- } \\
\text { to propio, de las posibilidades de éxito y } \\
\text { del grado de control sobre los sucesos y } \\
\text { las situaciones. }\end{array}$ \\
\hline llusión de control & $\begin{array}{l}\text { Mantener una expectación de éxito per- } \\
\text { sonal superior a lo que la probabilidad } \\
\text { objetiva garantiza. }\end{array}$ & $\begin{array}{l}\text { Conduce a sobrestimar el grado de con- } \\
\text { trol sobre los resultados de un curso de } \\
\text { acción y el grado en que las habilidades } \\
\text { propias influirán en los resultados. }\end{array}$ \\
\hline $\begin{array}{l}\text { llusión de } \\
\text { optimismo }\end{array}$ & $\begin{array}{l}\text { Tendencia a mantener un optimismo } \\
\text { irrealista en la determinación de las posi- } \\
\text { bilidades de experimentar resultados fu- } \\
\text { turos favorables. }\end{array}$ & $\begin{array}{l}\text { Conduce a sobrestimar la probabilidad } \\
\text { de los resultados futuros favorables. }\end{array}$ \\
\hline $\begin{array}{l}\text { llusión de } \\
\text { intensificación } \\
\text { personal }\end{array}$ & $\begin{array}{l}\text { Tendencia a sobrestimar las capacida- } \\
\text { des y habilidades personales propias } \\
\text { frente a las de los demás. }\end{array}$ & $\begin{array}{l}\text { Conduce a sobrestimar el grado de con- } \\
\text { trol sobre los resultados de un curso de } \\
\text { acción y el grado en que las habilidades } \\
\text { propias influirán en los resultados. }\end{array}$ \\
\hline $\begin{array}{l}\text { Percepción } \\
\text { selectiva }\end{array}$ & $\begin{array}{l}\text { En la aprehensión del contexto exterior, } \\
\text { las creencias y esquemas actúan como } \\
\text { filtro de la percepción. }\end{array}$ & $\begin{array}{l}\text { Las creencias y expectativas pueden } \\
\text { sesgar la observación de variables rele- } \\
\text { vante en los problemas. }\end{array}$ \\
\hline
\end{tabular}


Heurísticos y sesgos cognitivos en la dirección de empresas: un meta-análisis

Urra Urbieta, José Anastasio; Medina Lorza, Aida y Acosta Naranjo, Alejandro

Principales heurísticos y sesgos cognitivos y sus efectos
sobre la adopción de decisiones

Fuente: Elaboración propia (2010).

evitar las consecuencias negativas como la ansiedad y la depresión que acompañan la percepción de no mantener el control (Langer, 1975), y generar una mayor autoestima (Langer, 1994), lo que Menguzzato \& Urra (2004) ven como una causa legítima de la necesidad de los sujetos decisores de creer en nuestras habilidades; y, 2) los factores de habilidad o capacidad y los factores aleatorios están tan estrechamente asociados en la experiencia de las personas que a menudo tenemos verdaderas dificultades para discriminar entre unos y otros (Langer, 1975).

Finalmente, Nisbett \& Ross (1980) discuten sobre los heurísticos y los sesgos cognitivos a los que aquéllos conducen, las reglas normativas de inferencia, los factores emotivos y los factores cognitivos subyacentes en los sesgos o errores de inferencia, los costes personales y sociales de los sesgos humanos de inferen- cia, y las posibilidades de mejora de los procesos de inferencia.

En síntesis, la investigación hasta la fecha permite ofrecer una acotación del concepto de heurísticos cognitivos como procesos normativos simplificadores de selección, procesamiento y ajuste de la información, que conducen a sesgos de valoración y predicción, entendiéndose éstos desde su connotación negativa o de desviación ${ }^{1}$. Además, tales heurísticos y sesgos cognitivos parecen ser interculturales y resistentes al conocimiento, tal como demostraremos con una sencilla prueba empírica realizada en dos países a partir de una muestra de universitarios y directivos.

\section{Heurísticos y sesgos cognitivos en la dirección de empresas}

A partir del concepto de Estrategia Emergente de Mintzberg (1998), y tras la

1 Schwenk (1984) señala que donde algunas aportaciones utilizan el término "sesgos cognitivos" otras usan el de "heurísticos cognitivos" porque el primero sugiere que tales procesos generalmente tienen un impacto negativo sobre las decisiones. Sin embargo, como se ha discutido, las aportaciones de la psicología son claras en cuanto a la distinción entre ambos conceptos: los heurísticos cognitivos son ciertas normas simplificadoras de selección y procesamiento de la información que conducen a determinados sesgos de valoración y predicción (Tversky \& Kahneman, 1974). 
explicitación y formalización de la estrategia como conjunto de decisiones estratégicas por Eisenhardt (1999), el denominador común de los trabajos que pueden adscribirse al estudio de los heurísticos y sesgos cognitivos en el campo de la Dirección de Empresas, con su base y fundamentos en las aportaciones de la Behavioral Decision Theory, viene siendo, en general, la consideración de los efectos de los heurísticos y sesgos cognitivos sobre las decisiones estratégicas y, por ende, sobre la estrategia. En este sentido, Mintzberg et al. (1998) señalan que es del interés de la academia circunscrita a la Escuela Cognitiva del pensamiento estratégico la forma en que los individuos simplificamos la información mediante el empleo de sesgos en la formulación e implantación de las estrategias organizativas. En esta misma línea, Weick et al. (2005) plantean que, mediante procesos retrospectivos de imágenes (interpretaciones) como mecanismo para disminuir la incertidumbre y mediante significados que tienen como base sesgos cognitivos compartidos individual o colectivamente, las personas racionalizamos lo que pensamos y hacemos en las organizaciones.

Ahora bien, en relación a la incertidumbre y la adopción de decisiones, Schwenk (1984) sugiere que cuando se enfrentan a decisiones estratégicas, los directivos de empresa alteran sus percepciones del entorno de manera que éste aparezca más cierto; y dado que el estado psicológico de incertidumbre relativa a una decisión importante es tremendamente doloroso, los sujetos decisores utilizamos procesos perceptivos que simplifican la situación de decisión. Así, los heurísticos y sesgos cognitivos estarían en el origen de los procesos de simplificación cognitiva en la adopción de decisiones estratégicas (Schwenk, 1984, 1988). Este hecho, que sin duda puede actuar como facilitador en el proceso de adopción de determinadas decisiones estratégicas, complejas y comprometidas por su misma naturaleza, puede ocasionar efectos devastadores ante la ausencia de medidas de previsión frente a los riesgos.

Como prueba de lo anterior, se pueden observar organizaciones que con frecuencia perseveran invirtiendo recursos en una solución "aparente", pese a la existencia de potente evidencia sobre su disfuncionalidad (Fernández, 2009). Para Ross \& Staw (1993), esa tendencia a continuar desarrollando acciones ineficaces cuando no es probable que se pueda revertir la situación insatisfactoria, esa "escalada del compromiso" con determinados cursos de acción, se apoya, entre otras, en determinantes psicológicas tales como: sesgar los hechos para apoyar decisiones anteriores, asumir más riesgos cuando una decisión se presenta en términos negativos (para recuperar pérdidas), e involucrarse de manera exagerada en un determinado proyecto. De esta manera, y por temor al fracaso (deterioro de la autoestima), las personas no prestamos atención a las señales de riesgo y continuamos (Fernández, 2009). Los efectos de los heurísticos y sesgos cognitivos en la dirección de empresas, han sido recogidos en diversos trabajos. Con el fin de ampliar la perspectiva y claridad, en el Cuadro 2 se presenta una síntesis de las principales aportaciones al estudio de los heurísticos y sesgos cognitivos dentro del campo de la dirección de empresas y de la estrategia. 
Heurísticos y sesgos cognitivos en la dirección de empresas: un meta-análisis Urra Urbieta, José Anastasio; Medina Lorza, Aida y Acosta Naranjo, Alejandro

\section{Cuadro 2}

\section{Principales aportaciones al estudio de los heurísticos y sesgos cognitivos dentro del campo de la dirección de empresas y de la estrategia}

\begin{tabular}{|c|c|c|c|}
\hline Fuentes & Objeto de estudio & Naturaleza & Principales resultados y/o conclusiones \\
\hline $\begin{array}{l}\text { Kiesler \& } \\
\text { Sproull (1982) }\end{array}$ & $\begin{array}{l}\text { Procesos de percep- } \\
\text { ción de los directivos } \\
\text { Proceso de formulación } \\
\text { de la estrategia }\end{array}$ & Teórico & $\begin{array}{l}\text { Proponen que la forma en que operan los procesos } \\
\text { de cognición social hace que el proceso de percep- } \\
\text { ción de los directivos sea propenso a determinados } \\
\text { heurísticos y a la ocurrencia de ciertos errores. }\end{array}$ \\
\hline $\begin{array}{l}\text { Starbuck \& } \\
\text { Milliken (1988) }\end{array}$ & \multirow{3}{*}{$\begin{array}{l}\text { Proceso de formulación } \\
\text { de la estrategia }\end{array}$} & Teórico & $\begin{array}{l}\text { Plantean que la percepción está sujeta a numerosos } \\
\text { filtros como el tiempo, los procesos de racionaliza- } \\
\text { ción, y distorsiones en función de distintos factores. }\end{array}$ \\
\hline $\begin{array}{l}\text { Makridakis } \\
(1990)\end{array}$ & & Teórico & $\begin{array}{l}\text { Plantea el papel de los prejuicios y limitaciones del } \\
\text { juicio en el establecimiento de pronósticos y la planifi- } \\
\text { cación en la formación de estrategias. }\end{array}$ \\
\hline $\begin{array}{l}\text { Schoemaker } \\
(1990)\end{array}$ & & Teórico & $\begin{array}{l}\text { Expone el papel de los heurísticos y sesgos cogniti- } \\
\text { vos en la racionalidad para la obtención de rentas } \\
\text { económicas en la empresa. }\end{array}$ \\
\hline $\begin{array}{l}\text { Lant, Milliken } \\
\text { \& Batra (1992) }\end{array}$ & & Empírico & $\begin{array}{l}\text { Establecen el papel mediador de los procesos de atri- } \\
\text { bución egoprotectores de los directivos en la influen- } \\
\text { cia de la performance pasada de la empresa sobre la } \\
\text { reorientación estratégica de la misma. }\end{array}$ \\
\hline Bukszar (1999) & \multirow{7}{*}{$\begin{array}{l}\text { Proceso de adopción } \\
\text { de decisiones estratégi- } \\
\text { cas }\end{array}$} & Teórico & $\begin{array}{l}\text { Propone un modelo donde el sesgo de retrospectiva } \\
\text { introduce rigidez y coarta la flexibilidad para la formu- } \\
\text { lación de estrategias. }\end{array}$ \\
\hline $\begin{array}{l}\text { Zapata \& Canet } \\
\text { (2009) }\end{array}$ & & Teórico & $\begin{array}{l}\text { Concluyen que los cambios en el diseño organizativo } \\
\text { no sólo corresponden al condicionamiento de las va- } \\
\text { riables contextuales, sino que también son el fruto de } \\
\text { los procesos, sesgos y mapas cognitivos que se dan } \\
\text { en las mentes de los agentes decisores. }\end{array}$ \\
\hline $\begin{array}{l}\text { Schwenk } \\
(1984)\end{array}$ & & Teórico & $\begin{array}{l}\text { Induce los efectos de algunos heurísticos y sesgos } \\
\text { cognitivos sobre las diferentes fases del proceso de } \\
\text { adopción de decisiones estratégicas. }\end{array}$ \\
\hline $\begin{array}{l}\text { Schwenk } \\
(1988)\end{array}$ & & Teórico & $\begin{array}{l}\text { Propone la Integración de la cognición en el proceso } \\
\text { de adopción de decisiones estratégicas. }\end{array}$ \\
\hline $\begin{array}{l}\text { Clapham \& } \\
\text { Schwenk } \\
(1991)\end{array}$ & & Empírico & $\begin{array}{l}\text { Establecen la relación entre el patrón de atribución } \\
\text { egoprotectora en el procesamiento de la información } \\
\text { por parte de los directivos y la performance empresa- } \\
\text { rial generalmente negativa. }\end{array}$ \\
\hline $\begin{array}{l}\text { Zajac \& } \\
\text { Bazerman } \\
(1991)\end{array}$ & & Teórico & $\begin{array}{l}\text { Presentan los errores de juicio de los sujetos deciso- } \\
\text { res, al considerar las decisiones de la competencia. }\end{array}$ \\
\hline $\begin{array}{l}\text { Russo \& } \\
\text { Schoemaker } \\
(1992)\end{array}$ & & Teórico & $\begin{array}{l}\text { Discuten las causas, coste y posibles remedios del } \\
\text { exceso de confianza en la adopción de decisiones, y } \\
\text { las posibles bondades de éste durante la implemen- } \\
\text { tación de las decisiones. }\end{array}$ \\
\hline
\end{tabular}




\section{Cuadro 2 (Continuación) \\ Principales aportaciones al estudio de los heurísticos y sesgos cognitivos dentro del campo de la dirección de empresas y de la estrategia.}

\begin{tabular}{|c|c|c|c|}
\hline Fuentes & Objeto de estudio & Naturaleza & Principales resultados y/o conclusiones \\
\hline $\begin{array}{l}\text { Amit \& } \\
\text { Schoemaker } \\
(1993)\end{array}$ & & Teórico & $\begin{array}{l}\text { Sugieren la Influencia de los sesgos cognitivos en las } \\
\text { decisiones de los directivos sobre los comportamien- } \\
\text { tos subóptimos, la imitabilidad imperfecta, y las ren- } \\
\text { tas organizativas. }\end{array}$ \\
\hline $\begin{array}{l}\text { Corner, Kinick } \\
\text { \& Keats (1994) }\end{array}$ & & Teórico & $\begin{array}{l}\text { Identifican el papel central de la percepción selectiva } \\
\text { y los sesgos cognitivos en las fases de atención y co- } \\
\text { dificación de la información, dentro del proceso orga- } \\
\text { nizativo de decisión. }\end{array}$ \\
\hline $\begin{array}{l}\text { Burke \& } \\
\text { Steensma } \\
(1998)\end{array}$ & & Teórico & $\begin{array}{l}\text { Plantean el papel mediador del exceso de confianza } \\
\text { y del sesgo de escalada del compromiso en la rela- } \\
\text { ción entre la experiencia funcional de los componen- } \\
\text { tes del equipo directivo decisor y la performance de la } \\
\text { empresa. }\end{array}$ \\
\hline $\begin{array}{l}\text { Das \& Teng } \\
\text { (1999) }\end{array}$ & & Teórico & $\begin{array}{l}\text { Plantean la incidencia de los sesgos cognitivos de } \\
\text { anclaje, de la exposición a alternativas limitadas, de } \\
\text { la insensibilidad a la probabilidad previa de los resul- } \\
\text { tados y de la ilusión de control sobre los modelos de } \\
\text { adopción de decisiones Racional, de Evitación de la } \\
\text { Incertidumbre, del Incrementalismo Lógico, del Polí- } \\
\text { tico y del de Papelera o Garbage Can. }\end{array}$ \\
\hline $\begin{array}{l}\text { Jones \& } \\
\text { Roelofsma } \\
(2000)\end{array}$ & & Teórico & $\begin{array}{l}\text { Proporcionan una perspectiva sobre cómo afectan } \\
\text { los sesgos del falso consenso, el groupthinking, la } \\
\text { polarización de grupo y la escalada del compromiso } \\
\text { en el proceso de adopción de decisiones en equipo, y } \\
\text { sobre cómo dichos sesgos son promovidos por la } \\
\text { presión del tiempo y los altos niveles de incertidum- } \\
\text { bre. }\end{array}$ \\
\hline $\begin{array}{l}\text { Hodgkinson, } \\
\text { Maule, Bown, } \\
\text { Pearman \& } \\
\text { Glaister (2002) }\end{array}$ & & Teórico & $\begin{array}{l}\text { Ratifican las bondades del uso del mapeado causal } \\
\text { como una ayuda para la adopción de decisiones es- } \\
\text { tratégicas, al mostrar que dicha técnica ayuda a con- } \\
\text { trarrestar los efectos perniciosos de los heurísticos y } \\
\text { sesgos cognitivos. }\end{array}$ \\
\hline Urra (2009) & & Teórico & $\begin{array}{l}\text { Concluye que la función de los directivos no debe li- } \\
\text { mitarse a tomar decisiones sino que ha de extender- } \\
\text { se a intentar controlar los procesos de adopción de } \\
\text { decisiones para, a través de ellos, vigilar el proceso } \\
\text { de formación de la estrategia de la empresa. Propone } \\
\text { un modelo del proceso de adopción de decisiones } \\
\text { que integra los modelos del Actor Racional Único, Or- } \\
\text { ganizativo, Político, y Anárquico o Garbage Can. }\end{array}$ \\
\hline
\end{tabular}


Heurísticos y sesgos cognitivos en la dirección de empresas: un meta-análisis

Urra Urbieta, José Anastasio; Medina Lorza, Aida y Acosta Naranjo, Alejandro

\section{Cuadro 2 (Continuación) Principales aportaciones al estudio de los heurísticos y sesgos cognitivos dentro del campo de la dirección de empresas y de la estrategia.}

\begin{tabular}{l|}
\hline Fuentes \\
\hline March \& \\
Shapira (1987) \\
Kahneman \& \\
Lovallo (1993) \\
\hline
\end{tabular}

Krueger \& Dickson (1994)

Busenitz
(1999)

Cooper, Woo

\& Dunkelberg (1988)

Decisiones de emprendimiento

Objeto de estudio Naturaleza

Valoración del riesgo en Teórico la adopción de decisiones estratégicas
Shaver \& Scott (1991)

Palich \& Bagby (1995)

Teórico

Empírico

(Es)

\section{Principales resultados y/o conclusiones}

Plantean las diferencias entre la perspectiva del riesgo que mantienen los directivos y las mantenidas por las teorías normativas de la decisión, debido a que, en general, los directivos son insensibles a las estimaciones de probabilidad de los posibles resultados, a que sus decisiones se ven afectadas por el modo en que se centra su atención en los niveles críticos de performance, y a que establecen una fuerte distinción entre la asunción de riesgos y las "apuestas".

Discuten las implicaciones de los heurísticos y sesgos cognitivos en los procesos de adopción de decisiones organizativos, y señalan que resultan más perniciosos los sesgos que conducen a decisiones arriesgadas que los que propician comportamientos conservadores frente al riesgo.

Establecen la percepción de más oportunidades y la asunción de más riesgo por parte de los sujetos en los que se inducen sentimientos de autoconfianza en las capacidades propias, y se evidencia el sesgo por el que las expectativas de los resultados de la decisión dependen de las valoraciones previas de los mismos.

Empírico Establece que la asunción de riesgos por parte de los emprendedores puede ser explicada porque éstos son más proclives a los sesgos de representatividad y al exceso de confianza, lo que les lleva a percibir menos riesgo en las situaciones de decisión.

Empírico Establecen que la percepción optimista de los emprendedores sobre sus posibilidades de éxito se produce por la tendencia de los sujetos decisores a sostener e inflar el atractivo de una opción una vez que ésta ha sido seleccionada, y a creer que pueden controlar su propio destino.

Teórico Presentan un enfoque integrador que explica cómo la dependencia del comportamiento individual del emprendedor es contingente sobre los rasgos personales, los procesos cognitivos y el contexto externo.

Empírico Determinan que los emprendedores contemplan las situaciones arriesgadas de forma más optimista debido a que están más expuestos a ciertos sesgos cognitivos asociados con el exceso de confianza; ya que no se encuentran diferencias significativas entre aquéllos y los sujetos no emprendedores en cuanto a su propensión o aversión al riesgo. 


\section{Cuadro 2 (Continuación) Principales aportaciones al estudio de los heurísticos y sesgos cognitivos dentro del campo de la dirección de empresas y de la estrategia.}

\begin{tabular}{l|}
\hline Fuentes \\
\hline Busenitz \& \\
Barney (1997) \\
\hline \\
\hline
\end{tabular}

Simon,

Houghton

\& Aquino (1999)

Bryant (2007)

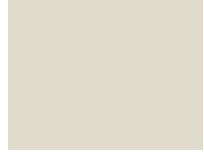

Duhaime

Decisiones de
\& Schwenk

(1985)

Jemison \&

Sitkin (1986)

Lyles (1987)

Alianzas

Estratégicas

Decisiones de adquisición y desinversión

Schwenk (1994)

Barr, Bogner, Golden-Biddle, Rao \& Thomas (1997)

Menguzzato \& Urra (2004)

\section{Naturaleza Principales resultados y/o conclusiones}

Empírico

Establecen una mayor susceptibilidad en los emprendedores, frente a los directivos de organizaciones grandes, al empleo de los heurísticos de representatividad y a los efectos de los sesgos que generan el exceso de confianza durante la adopción de decisiones.

Empírico Establecen que la percepción alterada del riesgo en la decisión estratégica de iniciar un nuevo negocio o empresa se produce por la concurrencia del exceso de confianza, la ilusión de control, y la ley de los "pequeños" números. Además, proponen que los sesgos cognitivos actúan como facilitadores emocionales de la decisión.

Empírico Sugiere que la heurística usada mediante el empleo de habilidades de autorregulación posibilita a los empresarios el desempeño en ambientes dinámicos y de incertidumbre, permitiéndoles evaluar y explotar efectivamente oportunidades a pesar de las presiones del límite de tiempo e información.

Teórico Plantean los efectos simplificadores de algunos sesgos cognitivos en los procesos de adquisición y desinversión empresariales.

Teórico Inducen el papel bloqueador del sesgo de "escalada del compromiso" en los procesos de adquisición, en el que dicho sesgo puede operar en contra del buen desarrollo de la integración.

Empírico Concluye que algunos errores en las distintas fases de desarrollo de las joint-ventures condicionan el aprendizaje organizativo, y que son ocasionados por las deficiencias de desarrollo cognitivo, bien por la falta de comprensión de la situación o bien por la rápida evolución de la misma.

Teórico Plantean la incidencia de algunos heurísticos y sesgos cognitivos en el desarrollo de las fases iniciales de formación de las Alianzas Estratégicas.

Teórico Plantean y argumentan los efectos de la simplificación cognitiva que puede tener lugar durante el desarrollo de las Alianzas Estratégicas sobre la performance de las mismas.

Teórico Presentan un modelo, con un papel central de una serie de heurísticos y sesgos cognitivos, en el que explican la realidad de los procesos intraorganizativos de adopción de decisiones en las fases iniciales de la formación de Alianzas Estratégicas, 
Heurísticos y sesgos cognitivos en la dirección de empresas: un meta-análisis Urra Urbieta, José Anastasio; Medina Lorza, Aida y Acosta Naranjo, Alejandro

\section{Cuadro 2 (Continuación) Principales aportaciones al estudio de los heurísticos y sesgos cognitivos dentro del campo de la dirección de empresas y de la estrategia.}

\begin{tabular}{|c|c|c|c|}
\hline Fuentes & Objeto de estudio & Naturaleza & Principales resultados y/o conclusiones \\
\hline $\begin{array}{l}\text { Thomas, Eden, } \\
\text { Hitt, \& Miller } \\
(2007)\end{array}$ & $\begin{array}{l}\text { Decisiones de } \\
\text { internacionalización }\end{array}$ & Empírico & $\begin{array}{l}\text { Establecen el papel pernicioso que juegan los ses- } \\
\text { gos cognitivos de percepción selectiva, y anclaje y } \\
\text { ajuste, sobre las decisiones de una empresa en rela- } \\
\text { ción a la entrada en nuevos mercados internaciona- } \\
\text { les. }\end{array}$ \\
\hline $\begin{array}{l}\text { Wickham } \\
\text { (2003) }\end{array}$ & $\begin{array}{l}\text { Decisiones } \\
\text { financieras }\end{array}$ & Empírico & $\begin{array}{l}\text { Establece que pese al conocimiento que se tenga so- } \\
\text { bre una materia y al uso de reglas practicas, el heu- } \\
\text { rístico de representatividad podría entorpecer la cali- } \\
\text { dad de las decisiones empresariales, especialmente } \\
\text { las relativas a la inversión en nuevas empresas. }\end{array}$ \\
\hline $\begin{array}{l}\text { Schwartz } \\
(2004)\end{array}$ & & Empírico & $\begin{array}{l}\text { Concluye que es necesario mejorar la forma en que } \\
\text { se aprecia la valía de la racionalidad en las decisio- } \\
\text { nes pasadas y la forma racional de proceder en el fu- } \\
\text { turo, para que el uso de los heurísticos sea consisten- } \\
\text { te con un proceso racional de adopción de decisiones } \\
\text { financieras. }\end{array}$ \\
\hline $\begin{array}{l}\text { Miller \& Saphira } \\
(2004)\end{array}$ & & Empírico & $\begin{array}{l}\text { Demuestran, mediante evidencia sobre desviacio- } \\
\text { nes sistemáticas de los valores de compra espera- } \\
\text { dos, la incidencia perniciosa de los heurísticos cogni- } \\
\text { tivos en las evaluaciones subjetivas de venta de ac- } \\
\text { ciones, y sugieren pautas para desarrollar una teoría } \\
\text { real de venta. }\end{array}$ \\
\hline Korte (2003) & $\begin{array}{l}\text { Decisiones para el } \\
\text { desarrollo de } \\
\text { recursos humanos }\end{array}$ & Teórico & $\begin{array}{l}\text { Plantea que para lograr un proceso de adopción de } \\
\text { decisiones riguroso referido al desarrollo de recursos } \\
\text { humanos, se debe incluir un examen explícito y retar } \\
\text { las hipótesis y sesgos que subyacen al proceso y a la } \\
\text { prescripción, para mitigar los efectos negativos de } \\
\text { orientaciones diversas producto de las característi- } \\
\text { cas de los accionistas, de los decisores y de los ana- } \\
\text { listas mientras interactúan para definir un problema y } \\
\text { generar una solución. }\end{array}$ \\
\hline
\end{tabular}

Fuente: Elaboración propia (2010).

Como se desprende de la síntesis recogida en el Cuadro 2, lo reciente del estudio del papel de los procesos cognitivos en la dirección de empresas y en la estrategia se plasma en ciertas peculiaridades que caracterizan a los trabajos que conforman este campo de investigación.

Así, en primer lugar, la naturaleza de los trabajos adscritos a este campo es, en general, predominantemente teórica. Sin embargo, en las dos últimas décadas la mayor madurez y elaboración de las bases teóricas se ha traducido en un incremento de las aportaciones empíricas sobre el papel de los heurísticos y sesgos cognitivos en la estrategia empresarial. No obstante, los estudios empíricos continúan siendo comparativamente esca- 
sos frente a las aportaciones teóricas y la evidencia derivada de la casuística.

En segundo lugar, como extensión de la tradición que configura sus raíces, el objeto de estudio de los trabajos que conforman este campo de investigación ha estado centrado, fundamentalmente y salvo excepciones, en los procesos de adopción de decisiones estratégicas. En este sentido, cabe señalar que, sin salirse de tal objeto de estudio, las excepciones se han centrado más en el análisis y discusión de la percepción de los ejecutivos per se. En cuanto al nivel de análisis, a partir de una unidad de análisis individual o de grupo, la mayor parte de los trabajos se han extendido a un nivel de análisis organizativo, lo que introduce la controvertida cuestión del vínculo entre las decisiones organizativas y la performance organizativa.

En tercer lugar, a pesar de que la gran mayoría de los trabajos se han centrado en los procesos de adopción de decisiones estratégicas como objeto de estudio, como consecuencia de la consideración subyacente en todos ellos de la estrategia como conjunto de decisiones estratégicas, los tópicos de interés difieren sustancialmente entre los trabajos. Dichos tópicos apuntan principalmente en varias direcciones: identificación de los diferentes factores que favorecen la ocurrencia de los heurísticos, los que, a su vez, afectan la percepción de los directivos; papel que representan algunos procesos de simplificación cognitiva y sesgos en la racionalidad para la formulación de estrategias, y sus consecuencias en la performance de la empresa; propuestas de modelos del proceso de adopción de decisiones estratégicas de grupos e individuos, en los que se incluyen algunos heurísticos y sesgos cognitivos, y su incidencia en dichos procesos; efectos de algunos heurísticos y sesgos cognitivos sobre la percepción del riesgo en los sujetos decisores, y sus repercusiones en la adopción de decisiones; participación y efectos de algunos heurísticos y sesgos cognitivos en la adopción de decisiones de emprendimiento; efectos de algunos sesgos cognitivos en la adopción de decisiones de desarrollo de recursos humanos, y de adquisición y desinversión; incidencia de algunos sesgos cognitivos, y su modulación a partir de ciertos factores de contingencia, en la ocurrencia de errores en alguna de las fases de las Alianzas Estratégicas y en la performance de las mismas; incidencia de algunos heurísticos y sesgos cognitivos en la adopción de decisiones sobre la entrada o no de una empresa en mercados internacionales. Ahora bien, sin duda debido al catalizador financiero de la crisis, y a la inestabilidad e incertidumbre internacional en que ésta ha devenido, desde 2003, en los últimos años predominan los estudios de los heurísticos y sesgos cognitivos en campos como las finanzas y las decisiones de carácter financiero, la política, particularmente internacional, y el riesgo y su evaluación.

En cuarto lugar, respecto de la evidencia empírica aportada por esta corriente de trabajos, podemos constatar que los trabajos de campo constituyen la excepción más que la regla. En este sentido, la mayor parte de la evidencia empírica se deriva de experimentos de laboratorio (Krueger \& Dickson, 1994; Simon et al., 1999), o de investigaciones con un diseño marcadamente experimental (Busenitz \& Barney, 1997; Palich \& Bagby, 
Heurísticos y sesgos cognitivos en la dirección de empresas: un meta-análisis Urra Urbieta, José Anastasio; Medina Lorza, Aida y Acosta Naranjo, Alejandro

1995; Busenitz, 1999; Wickham, 2003; Miller \& Saphira, 2004).

Finalmente, aunque algunos trabajos señalan que los heurísticos y sesgos cognitivos podrían tener efectos beneficiosos durante la formulación de las decisiones estratégicas (Busenitz, 1999; Simon et al., 1999; Menguzzato \& Urra, 2004; Zapata y Canet, 2009), o incluso durante la propia implementación de las mismas (Russo \& Schoemaker, 1992; Simon et al., 1999; Bryant, 2007), la idea común y recurrente que las conclusiones, tanto teóricas como empíricas, de los trabajos de esta corriente de literatura sugieren es la del carácter pernicioso de los efectos derivados de los heurísticos y de los sesgos cognitivos. Entre estos efectos destaca la alteración de la percepción del riesgo inherente a las situaciones de decisión, siendo esta percepción el factor principal con efectos directos sobre la elección final (decisión adoptada). Aunque, hasta donde sabemos, tales ideas permanecen pendientes de un mayor contraste empírico, sí parece claro que si bien los heurísticos son no suprimibles por su naturaleza humana intrínseca, por su carácter de procesos podrían ser incididos junto a sus efectos (sesgos).

\section{La gestión de los procesos de decisión}

Sin duda, una de las mayores tautologías en el campo de la dirección de empresas es la que se refiere a la evaluación, o juicio, de las decisiones a partir de sus resultados: "tal decisión fue buena, o mala, porque sus resultados fueron buenos, o malos". Juzgar las decisiones de esta forma conlleva, por un lado, obviar los procesos que llevan a la adopción de las mismas, y por otro, pasar por alto la incidencia del entorno sobre tales procesos y sobre el desarrollo de las propias decisiones. Además, resulta en juicios estériles, pues introduce un determinismo de acción que impide el análisis y la gestión de los procesos mediante los que las decisiones se adoptan.

Consecuentemente, equiparar la propia estrategia con un proceso global de adopción de decisiones estratégicas interrelacionadas, como ya apuntamos, conlleva asumir dos ideas básicas, pero fundamentales, respecto a la propia adopción de decisiones, a saber: 1) que procesos diferentes generan elecciones diferentes, y 2) que diferentes elecciones generan resultados diferentes.

La primera asunción, que los procesos de adopción de decisiones están relacionados con la elección efectuada, puede no resultar tan obvia si consideramos que las constricciones del entorno juegan un papel clave determinando las alternativas $\mathrm{y}$, por tanto, reducen la importancia de los procesos de elección (Hannan \& Freeman, 1977; Pfeffer \& Salancik, 1978; DiMaggio \& Powell, 1983). Sin embargo, algunos autores han argumentado que, a pesar de las constricciones impuestas por el entorno, los directivos mantienen un grado significativo de control sobre las elecciones posibles (Schoemaker, 1990; Oliver, 1991, 1997; Child, 1997). Lo cierto es que una extensa casuística pone de manifiesto que, sometidos a las mismas circunstancias externas, algunos directivos realizan elecciones con consecuencias devastadoras mientras que otros realizan elecciones de la misma naturaleza con consecuencias 
más benignas. Dado que esta variación en las decisiones no parece tener su origen en las constricciones externas, es más que probable que las elecciones dependan en gran medida del proceso de adopción de decisiones seguido.

Respecto de la segunda asunción que apuntábamos, que la elección realizada influye sobre los resultados, de nuevo diferentes argumentos dejan pocas dudas de que las fuerzas externas también influyen sobre los resultados de la elección realizada (Pfeffer \& Salancik, 1978; DiMaggio \& Powell, 1983). En este sentido, también una extensa casuística pone de manifiesto que cambios repentinos en las estrategias de los competidores o en la demanda han convertido en desastre determinadas elecciones $\mathrm{o}$, a la inversa, las han convertido en éxitos rotundos. Sin embargo, dado que resulta difícil pensar en una decisión en la que todas las posibles alternativas tengan las mismas probabilidades de éxito o fracaso, más bien parece poco probable que las contingencias externas eliminen totalmente la influencia de la elección efectuada sobre su eficacia.

Dado que ambas asunciones resultan plausibles, es razonable esperar que los procesos de adopción de decisiones influyan en la eficacia de las decisiones, de manera que estos procesos permitan a los decisores anticipar con mayor precisión los factores externos y realizar elecciones más exitosas que aquéllos que no sigan esta dinámica. Es más, el papel de la discreción directiva ante la creciente turbulencia del entorno radica fundamentalmente en buscar cursos de acción viables frente a las constricciones del entorno (Schoemaker, 1990; Amit \& Schoema- ker, 1993), siendo ésta, precisamente, la esencia de los sistemas de gestión, la esencia de la Dirección de Empresas.

Por otra parte, una de las características más centrales de las decisiones estratégicas es su carencia de estructura (Schwenk, 1984). El carácter casi unánime de la extensa literatura sobre este punto permite afirmar que, casi por definición y tratándose de una cuestión de grado, las decisiones estratégicas se caracterizan por su naturaleza idiosincrásica y por su falta de estructura. Por tanto, es común en ellas la carencia de una solución calculable mediante algoritmos matemáticos, su gran complejidad e incertidumbre, sus efectos a largo plazo y, por tanto, su carácter difícilmente reversible a corto y medio plazo.

Basándose implícitamente en el concepto de racionalidad de proceso, y centrándose en los límites de la capacidad individual de procesamiento de información, Galbraith (1973) ya sugería que las organizaciones deben procesar más información en la medida que aumentan la complejidad e incertidumbre de sus tareas. Normalmente, las normas, planes y políticas suponen las guías iniciales para tratar los problemas, y representan el grado de preplanificación racional en el seno de la organización para la anticipación de problemas futuros; sin embargo, no son suficientes para afrontar todas las situaciones que puedan surgir (Schoemaker, 1993). Para Weick (1979), el énfasis está en la idea de que lo que ocurre en una organización fue esperado o planeado en algún momento de tiempo anterior. Además, dado que las distintas formas de racionalidad limitada inherentes al ser humano no resultan incompatibles con la ra- 
Heurísticos y sesgos cognitivos en la dirección de empresas: un meta-análisis Urra Urbieta, José Anastasio; Medina Lorza, Aida y Acosta Naranjo, Alejandro

cionalidad de proceso (Eisenhardt \& Zbaracki, 1992$)^{2}$, el reto cotidiano de los directivos se convierte en contribuir a que sus organizaciones desarrollen procedimientos que amortigüen frente a los efectos negativos de tales limitaciones durante los procesos de adopción de decisiones estratégicas.

Esta concepción de "racionalidad intencionada"3 ${ }^{3}$, ha devenido en una corriente de investigación que ha tratado de determinar cómo se adoptan las decisiones organizativas importantes y por qué se adoptan de la forma en que se hace. En esta corriente de investigación se enmarcan, entre otros, los trabajos de Fredrickson \& Laquinto (1989), Dean \& Sharfman (1993), Eisenhardt (1999), y Menguzzato \& Urra (2004).

Con sus diferencias, estos trabajos mantienen en común la idea general, que suscribimos, de que las decisiones organizativas difícilmente pueden ser racionales en el sentido de la Teoría de la Utilidad Subjetiva Esperada. Así, aunque pocas decisiones pueden ser racionales en el sentido de la Teoría Económica Clásica, la racionalidad de proceso, o el grado de recolección de información y el análisis de la misma, varía sustancialmente entre ellas (Dean \& Sharfman, 1993). Adicionalmente, el proceso de adopción e implementación, independientemente del contexto, influye en los resultados de las decisiones estratégicas, estando fuera de duda su papel fundamental en la relación con la eficacia de las mismas (Menguzzato \& Urra, 2004). Una comprensión más amplia del proceso genera decisiones más efectivas, aparentemente con una mayor efectividad en entornos estables que en entornos inestables (Menguzzato \& Urra, 2004).

Ahora bien, durante el proceso de adopción de decisiones estratégicas, el empleo por parte de los sujetos decisores de determinados heurísticos cognitivos perniciosos no es neutro. Esto es, existen ciertos factores de contingencia, configurables y controlables por la dirección por tanto, con efectos directos sobre el mayor o menor desarrollo de ciertos heurísticos cognitivos durante el proceso de adopción de decisiones. Estos factores de contingencia configuran, a partir de la gestión de los procesos de adopción de decisiones, la estructura del grupo decisor, la dinámica del grupo decisor, y determinados elementos institucionales.

La estructura del grupo decisor como factor de contingencia encuentra su base en las ideas de Hambrick \& Mason (1984). A partir de esta aportación se ha configurado un cuerpo de trabajos centrados en las implicaciones de la composición demográfica de los equipos di-

2 Como ya se ha apuntado, el propio Simon (1978) reconoce la racionalidad de proceso como el grado en el que el proceso de adopción de decisiones refleja la intención y los esfuerzos del sujeto decisor para realizar la mejor decisión posible.

3 Caracterizada por el intento contextualmente razonable de recolección de la información necesaria para la formación de las expectativas relativas a las diferentes alternativas y por el uso de esa información en la decisión final (Dean \& Sharfman, 1993). 
rectivos sobre el proceso de adopción de decisiones. Estos trabajos adoptan como premisa general que la composición demográfica del equipo es un indicador fuerte de su composición cognitiva y en valores, y que, por tanto, la diversidad demográfica del grupo resulta indicativa de su diversidad cognitiva. En este sentido, diferentes argumentos sugieren que la diversidad cognitiva del equipo decisor afecta al proceso de adopción de decisiones que desarrolle. Sin embargo, la evidencia empírica es aún inconclusa en cuanto al signo de tales efectos (Menguzzato \& Urra, 2004).

Como justificación del efecto positivo de la diversidad cognitiva sobre los procesos de adopción de decisiones, se argumenta que dicha diversidad tiende a crear desacuerdos cuando se tratan asuntos estratégicos (Lant et al., 1992), lo que permite una comprensión más amplia del proceso de adopción de decisiones (Miller et al., 1998) y la generación de diversas posturas frente a la cuestión a decidir, disminuyendo así la posibilidad del groupthink (Janis \& Mann, 1977) y del exceso de confianza (Burke \& Steensma, 1998). Otro argumento en este sentido está relacionado con los costes. Se sugiere, desde esta consideración, que el proceso de adopción de decisiones estratégicas incrementaría en su comprensión al reconciliar las posturas divergentes y lograr un acuerdo para la acción (Menguzzato \& Urra, 2004), mediante una mayor inversión de recursos en análisis, consultas y discusiones (Fredrickson \& Mitchell, 1984). Adicionalmente, aportaciones de la psicología social sugieren que la cohesión del equipo directivo decisor se verá influida de manera negativa por la diversidad cognitiva (Condon \& Crano, 1988), lo que a su vez, al propiciar el debate entre los integrantes del equipo decisor (Miller et al., 1998), aumenta la comprensión del proceso de adopción de decisiones y reduce las probabilidades del groupthink (Janis y Mann, 1977).

En cuanto a la justificación del efecto negativo de la diversidad cognitiva sobre los procesos de adopción de decisiones, se argumenta, por un lado, que la radicalidad en las posturas de los integrantes del equipo podría conducir a desacuerdos irreconciliables en las cuestiones a decidir (Lant et al., 1992; Miller et al., 1998). Por otro lado, y en relación a la comunicación y al carácter idiosincrático de ésta (Cyert \& March, 1963), la diversidad en las estructuras cognitivas de los integrantes del equipo decisor conlleva, en buena medida, diferencias en sus esquemas psicolingüísticos (Berger \& Luckmann, 1967), produciendo dificultades y bloqueos en la comunicación. Todo lo anterior llevaría a negociaciones segmentadas fuera del espacio de debate del equipo, disminuyendo así la discusión pública y transparente, $\mathrm{y}$, por ende, la comprensión del proceso de adopción de decisiones (Miller et al., 1998).

Desde estas consideraciones, en síntesis, la estructura del grupo que desarrolla el proceso de adopción de decisiones, que está estrechamente relacionada con elementos de la estructura organizativa formal e informal y se traduce en una mayor o menor diversidad cognitiva entre sus miembros, determina la variedad de puntos de vista con que se afronta un problema e incide sobre el uso de ciertos heurísticos cognitivos. 
Heurísticos y sesgos cognitivos en la dirección de empresas: un meta-análisis Urra Urbieta, José Anastasio; Medina Lorza, Aida y Acosta Naranjo, Alejandro

Como factor de contingencia, la dinámica del grupo se asienta en el planteamiento de que la calidad de las decisiones estratégicas influye en la performance de la empresa. Desde esta consideración, una línea de trabajo se ha centrado en los efectos de la dinámica del equipo directivo sobre el proceso de adopción de decisiones y sus resultados (Menguzzato \& Urra, 2004). La idea común en esta línea de trabajo radica en que la calidad de las decisiones estratégicas depende de la capacidad cognitiva del equipo directivo tanto como de su proceso de interacción para producir las decisiones (Amason, 1996).

Dentro de este cuerpo de trabajos se pueden distinguir dos posiciones. Un primer grupo ha analizado empíricamente los efectos comparativos de diferentes técnicas de interacción orientadas a potenciar la diversidad cognitiva del grupo a partir de la gestión del conflicto intragrupo (Schweinger et al., 1989; Cosier \& Schwenk, 1990; Murrel et al., 1993; Eisenhardt, 1999). Estos trabajos muestran que las técnicas de elevado conflicto incrementan la comprensión del proceso de adopción de decisiones, y sus consecuencias favorables sobre el proceso y sobre la calidad de la decisión; pero, al mismo tiempo, propician una mayor insatisfacción y un peor clima entre los miembros del equipo (Menguzzato \& Urra, 2004). Algunos investigadores, con respecto a estos resultados, cuestionan la posibilidad de alcanzar de manera simultánea calidad y consenso en la adopción de decisiones estratégicas (Eisenhardt \& Zbaracki, 1992).

Tratando de ofrecer respuesta al anterior trade off, un segundo conjunto de trabajos ha ahondado sobre los efectos del conflicto en los procesos grupales de adopción de decisiones estratégicas. La conclusión de esta línea de trabajo es que el conflicto es un constructo constituido, al menos, por dos dimensiones interrelacionadas: 1) conflicto concerniente a la tarea (Jehn, 1995, 1997), o conflicto cognitivo (Amason, 1996); y, 2) conflicto concerniente a las relaciones (Jehn, 1995, 1997) o conflicto afectivo (Amason, 1996). En cuanto al conflicto cognitivo, sus efectos en el desempeño del equipo decisor (Amason, 1996) dependen del tipo de tarea (Jehn, 1995). Con respecto al conflicto afectivo, independientemente del tipo de tarea que realice el equipo decisor, sus efectos sobre su desempeño son negativos (Jehn, 1995, 1997; Amason, 1996). Consecuentemente, la recomendación sería evitar el conflicto afectivo y promover el conflicto cognitivo entre el equipo decisor (Jehn, 1995, 1997; Amason, 1996). Sin embargo, ciertas cuestiones permanecen irresolutas (Menguzzato \& Urra, 2004): algunos resultados indican que no hay una relación lineal entre el rendimiento del grupo y el nivel del conflicto cognitivo, ya que los mejores resultados en el rendimiento del equipo decisor se producen ante niveles intermedios de conflicto cognitivo (Jehn, 1995); también se ha encontrado que, incluso cuando las tareas son no rutinarias, el conflicto cognitivo produce malestar en el grupo (Jehn, 1995); adicionalmente, es posible que el conflicto cognitivo, en la propia dinámica del equipo, derive en conflicto afectivo (Amason, 1996; Jehn, 1997), sin que se tengan conocimientos sobre tal dinámica (Menguzzato y Urra, 2004). 
En síntesis, la dinámica del grupo que desarrolla el proceso de adopción de decisiones, que se traduce en un mayor o menor grado de conflicto, tanto cognitivo como afectivo, entre sus miembros, determina la cohesión del grupo e incide sobre el uso de ciertos heurísticos cognitivos. Además, la dinámica del grupo se ve, a su vez, influida por la estructura del mismo; pues no basta una estructura de heterogeneidad si ésta no se dinamiza para que aflore, y ante niveles mínimos de heterogeneidad no hay dinámica posible que estimule el conflicto.

Otro de los factores de contingencia, más estructural sin duda, pero también susceptible de gestión, es el conformado por los elementos institucionales. Éstos encuentran su base en las teorías institucionales, por las que las organizaciones maniobran como una red social de relaciones en donde los valores, las normas y los estándares de comportamiento (DiMaggio \& Powell, 1983) condicionan las decisiones a criterios humanos construidos socialmente (Berger \& Luckmann, 1967; Weick et al., 2005), haciendo énfasis en un comportamiento organizativo irreflexivo y rutinario socialmente definido, distinto de la racionalidad propia de los enfoques económicos y estratégicos, preocupados por un comportamiento organizativo económicamente justificado (Oliver, 1997). Estas teorías asumen que los individuos son susceptibles a la influencia social y buscan la aprobación de sus comportamientos (Scott, 1987; Zucker, 1987), lo que incide directamente sobre el uso de ciertos heurísticos cognitivos por parte de los sujetos decisores.

Esta línea de pensamiento ha dado origen, también, a dos corrientes de estu- dio complementarias: la concerniente a los factores institucionales externos y la concerniente a los factores institucionales internos (Menguzzato \& Urra, 2004).

De acuerdo con la primera corriente, los elementos institucionales externos (competidores, universidades, legislaciones, entre otros) generan presiones sobre la adopción de decisiones organizativas (DiMaggio \& Powell, 1983), pudiendo propiciar procesos de adopción de decisiones irreflexivas, con una racionalidad económica limitada (Oliver, 1997).

En relación a segunda corriente, las organizaciones son el origen central de institucionalización porque, fundamentado en aspectos aceptados de la cotidianidad, se produce un proceso de conformidad que lleva a una institucionalización de nuevos elementos culturales (Zucker, 1987) que, al incorporarse a los objetivos organizativos, aumentan la eficacia de la organización (Zucker, 1987; Oliver, 1997).

Ahora bien, aunque las bases teóricas parecen claras (DiMaggio \& Powell, 1983), la evidencia empírica es escasa y en ocasiones poco clara (Menguzzato \& Urra, 2004).

Desde estas consideraciones, en síntesis, los elementos institucionales que hacen referencia a las normas, valores, hábitos, costumbres, rutinas y procedimientos operativos compartidos colectivamente por los miembros de la organización, y que la organización construye con el paso del tiempo a partir de las relaciones sociales internas y externas, inciden directamente sobre el uso de ciertos heurísticos cognitivos por parte de los sujetos decisores. 
Heurísticos y sesgos cognitivos en la dirección de empresas: un meta-análisis Urra Urbieta, José Anastasio; Medina Lorza, Aida y Acosta Naranjo, Alejandro

Finalmente, y como conclusión de este apartado, al igual que Starbuck \& Milliken (1988), Russo \& Schoemaker (1992), Bukszar (1999), Menguzzato \& Urra (2004) o Bryant (2007), pensamos que el mejor antídoto contra los efectos perniciosos de los heurísticos y sesgos cognitivos en los procesos organizativos de adopción de decisiones es conocerlos, ser conscientes de cómo actúan y de sus consecuencias, y gestionar los factores de contingencia bajo cuya influencia parecen encontrarse. Pero para esto, y dado que los heurísticos son conceptualmente abstractos, intangibles, inaprensibles e inefables, se requiere que dichos heurísticos y sus efectos sean enseñados y explicados mediante una estrategia didáctica interactiva, basada en experimentos y simulaciones de adopción de decisiones sencillas, como presentamos en una prueba empírica a continuación.

\section{Estrategia didáctica a través de una prueba empírica}

Como ejemplo de estrategia didáctica, a continuación presentamos una sencilla prueba empírica que evidencia la existencia, interculturalidad y resistencia al conocimiento de los heurísticos y sesgos cognitivos. Para tal fin, se han adaptado cuatro ejercicios de decisión de Makridakis $(1990)^{4}$, a saber: 1) \% de naciones africanas en la ONU; 2) ingenieros y abogados; 3) despedir 600 empleados; $y$,
4) identificar $n^{\circ}$ de F's. El objetivo para el ejercicio 1) consiste en determinar el sesgo de anclaje y ajuste; el ejercicio 2) trata de determinar el sesgo de confirmación y el heurístico de disponibilidad; en el 3), el objetivo es determinar la coherencia de nuestras preferencias frente al marco de la situación; y para el 4), el objetivo radica en determinar el sesgo de confianza y acierto.

Estos ejercicios se administran en las aulas, y allí también se explica su lógica y se describen los resultados, de manera que los alumnos mejoren su comprensión sobre los heurísticos y sesgos cognitivos al poder "verlos" y "tocarlos". Como se aprecia en el Cuadro 3, cada ejercicio cuenta con dos versiones diferenciadas.

La muestra se descompuso en dos partes simétricas, administrando a cada una de ellas una de las versiones completa de los 4 ejercicios. Los ejercicios fueron administrados, de esta forma, en Cali (Colombia) y Valencia (España) a sendas muestras estratificadas tal como se presenta en el Cuadro 4.

En cada país y en cada estrato de la muestra los grupos se dividieron en dos subgrupos simétricos. Al subgrupo 1 de cada estrato se le entrego la Versión 1 de los 4 ejercicios, y al subgrupo 2 de cada estrato la Versión 2. De esta manera, en la muestra completa se tuvo, aproximadamente, la mitad de Versiones 1 y la mitad de Versiones 2 en todos los estratos y en ambos países. La Tabla 1 presenta los

4 Por motivos de extensión y forma se omite el literal de los ejercicios en cuestión y una descripción detallada de los mismos; pueden encontrarse íntegramente en Makridakis (1990). 


\section{Cuadro 3}

Versiones 1 y 2 de los ejercicios de decisión

\begin{tabular}{|c|c|c|}
\hline & Versión 1 & Versión 2 \\
\hline Ejercicio 1 & $\begin{array}{l}\text { Estimar \% de naciones africanas en la ONU: va- } \\
\text { lor inicial al azar } 10 \%\end{array}$ & $\begin{array}{l}\text { Estimar \% de naciones africanas en la ONU: } \\
\text { valor inicial al azar } 65 \% \text {. }\end{array}$ \\
\hline Ejercicio 2 & $\begin{array}{l}\text { Ingenieros y abogados: composición muestra } \\
70 \% \text { Abogados y } 30 \% \text { Ingenieros. }\end{array}$ & $\begin{array}{l}\text { Ingenieros y abogados: composición muestra } \\
70 \% \text { Abogados y } 30 \% \text { Ingenieros + Información } \\
\text { adicional irrelevante. }\end{array}$ \\
\hline Ejercicio 3 & $\begin{array}{l}\text { Despedir } 600 \text { empleados: preferencias ganan- } \\
\text { cia (A = salvar } 200 \text { puestos). }\end{array}$ & $\begin{array}{l}\text { Despedir } 600 \text { empleados: preferencias pérdida } \\
\text { ( } A=\text { despedir } 400 \text { personas). }\end{array}$ \\
\hline Ejercicio 4 & Identificar nº de F's en una sentencia: leer 1 vez. & Identificar nº de F's: sin restricción de lectura. \\
\hline
\end{tabular}

Fuente: Elaboración propia.

Cuadro 4

Estratificación de la muestra Colombia-España

\begin{tabular}{lllc}
\hline \multicolumn{2}{c}{ Estratificación Colombia } & \multicolumn{2}{c}{ Estratificación España } \\
\hline Pregrado & 45 sujetos & 1er $\mathbf{y ~} \mathbf{2}^{\circ}$ Ciclo & 35 sujetos \\
Directivos & 41 sujetos & Doctorado/Directivos & 15 sujetos \\
MBA & 47 sujetos & MBA/Directivos & 21 sujetos \\
TOTAL & $\mathbf{1 3 3}$ sujetos & TOTAL & $\mathbf{7 1 ~ s u j e t o s ~}$
\end{tabular}

Fuente: Elaboración propia.

resultados de los estadísticos descriptivos asociados a las muestras española y colombiana en las dos versiones de los 3 primeros ejercicios. Como puede observarse, las medias son distintas en cada versión, evidenciando los heurísticos y sesgos cognitivos que los ejercicios tratan de determinar.

Sin embargo, cabe la posibilidad de que tales diferencias de respuesta entre ambas versiones de los ejercicios fueran debidas al mero azar. Para contrastar esta hipótesis aplicamos la prueba para muestras independientes a los datos correspondientes a los ejercicios 1, 2 y 3 en la muestra colombiana y española. Utilizamos el paquete SPSS, que utiliza el algoritmo de la prueba de Levene y de la "t de Student" para contrastar la diferencia en varianzas y medias entre ambas submuestras (Versión 1 y Versión 2). Para el análisis de los datos correspondientes al ejercicio 4 se utilizaron tablas de contingencia de doble entrada: $N^{\circ}$ de F's e Intervalo de Confianza. Los resultados de estos análisis se sintetizan en las Tablas 2 y 3.

Tal como reflejan la prueba de Levene y la "t de Student", la Versión 1 y la Versión 2 son muestras distintas e independientes (varianzas y medias significativamente distintas) sin intervención del azar. Por tanto, si las diferencias de respuesta entre la Versión 1 y la Versión 2 no tienen su origen en el azar, sólo pueden tenerlo por el efecto de los heurísticos y sesgos cognitivos que tratan de determinar. 
Heurísticos y sesgos cognitivos en la dirección de empresas: un meta-análisis Urra Urbieta, José Anastasio; Medina Lorza, Aida y Acosta Naranjo, Alejandro

Tabla 1

Resultados Versión 1-Versión 2 España-Colombia

\begin{tabular}{|c|c|c|c|c|c|c|c|}
\hline \multirow{2}{*}{\multicolumn{2}{|c|}{ Versión }} & \multicolumn{3}{|c|}{ Colombia } & \multicolumn{3}{|c|}{ España } \\
\hline & & Ejercicio 1 & Ejercicio 2 & Ejercicio 3 & Ejercicio 1 & Ejercicio 2 & Ejercicio 3 \\
\hline \multirow{4}{*}{ v 1} & Media & $(\%$ Af. $=21,3)$ & $(25 \% \mathrm{I} / 75 \% \mathrm{~A})$ & $(47 \%$ B/53\% A) & $(\% A f .=25,4)$ & $(20 \% \mathrm{l} / 80 \% \mathrm{~A})$ & $(49 \% B / 51 \% A)$ \\
\hline & & 21,33 & 25 & 47 & 25,40 & ,20 & ,49 \\
\hline & N & 72 & 73 & 73 & 35 & 35 & 35 \\
\hline & Desv. típ. & 21,866 & ,434 &, 502 & 19,994 & ,406 &, 507 \\
\hline \multirow{4}{*}{ v 2} & Media & $(\% A f .=36,4)$ & $(67 \% 1 / 33 \% A)$ & $(58 \% \mathrm{~B} / 42 \% \mathrm{~A})$ & $(\%$ Af. $=35,9)$ & $(44 \% 1 / 56 \% A)$ & $(64 \% B / 36 \% A)$ \\
\hline & & 36,42 &, 67 &, 58 & 35,89 &, 44 &, 64 \\
\hline & N & 60 & 60 & 60 & 36 & 36 & 36 \\
\hline & Desv. típ. & 24,196 & ,475 & ,497 & 24,469 &, 504 & ,487 \\
\hline Total & $\mathrm{N}$ & 132 & 133 & 133 & 71 & 71 & 71 \\
\hline
\end{tabular}

Fuente: Elaboración propia.

Tabla 2

Prueba de muestras independientes Versión 1-Versión 2 Colombia-España

\begin{tabular}{|c|c|c|c|c|c|c|c|c|c|c|}
\hline \multirow{3}{*}{\multicolumn{2}{|c|}{$\begin{array}{c}\text { N1 = VERSIÓN } 1 \\
\text { N2 = VERSIÓN } 2 \\
\text { Colombia }\end{array}$}} & \multicolumn{2}{|c|}{$\begin{array}{c}\text { Prueba de } \\
\text { Levene para la } \\
\text { igualdad } \\
\text { de varianzas }\end{array}$} & \multicolumn{7}{|c|}{ Prueba t para la igualdad de medias } \\
\hline & & \multirow[b]{2}{*}{$\mathbf{F}$} & \multirow[b]{2}{*}{$\begin{array}{l}\text { P } \\
\text { valor } \\
\text { Sig. }\end{array}$} & \multirow[b]{2}{*}{$t$} & \multirow[b]{2}{*}{ gl } & \multirow[b]{2}{*}{$\begin{array}{l}\text { P valor } \\
\text { Sig.(bil } \\
\text { ateral) }\end{array}$} & \multirow[b]{2}{*}{$\begin{array}{l}\text { Dife- } \\
\text { rencia } \\
\text { de } \\
\text { medias }\end{array}$} & \multirow[b]{2}{*}{$\begin{array}{l}\text { Error } \\
\text { típ. de } \\
\text { la dife- } \\
\text { rencia }\end{array}$} & \multicolumn{2}{|c|}{$\begin{array}{l}95 \% \text { Intervalo de } \\
\text { confianza para la } \\
\text { diferencia }\end{array}$} \\
\hline & & & & & & & & & Inferior & Superior \\
\hline \multirow[t]{2}{*}{$\begin{array}{l}\text { Ejercicio } \\
1\end{array}$} & $\begin{array}{l}\text { Se han asu- } \\
\text { mido varian- } \\
\text { zas iguales }\end{array}$ & 4,218 & ,042 & $-3,759$ & 130 &, 000 & $-15,083$ & 4,012 & $-23,021$ & $-7,146$ \\
\hline & $\begin{array}{l}\text { No se han } \\
\text { asumido va- } \\
\text { rianzas } \\
\text { iguales }\end{array}$ & & & $-3,725$ & 120,325 & ,000 & $-15,083$ & 4,049 & $-23,101$ & $-7,066$ \\
\hline \multirow[t]{2}{*}{$\begin{array}{l}\text { Ejercicio } \\
2\end{array}$} & $\begin{array}{l}\text { Se han asu- } \\
\text { mido varian- } \\
\text { zas iguales }\end{array}$ & 4,616 & 034 & $-5,321$ & 131 &, 000 &,- 420 & ,079 &,- 576 &,- 264 \\
\hline & $\begin{array}{l}\text { No se han } \\
\text { asumido va- } \\
\text { rianzas } \\
\text { iguales }\end{array}$ & & & $-5,273$ & 120,995 & ,000 &,- 420 & ,080 &,- 578 &,- 262 \\
\hline
\end{tabular}


Tabla 2 (Continuación)

Prueba de muestras independientes Versión 1-Versión 2 Colombia-España

\begin{tabular}{|c|c|c|c|c|c|c|c|c|c|c|}
\hline \multirow{3}{*}{\multicolumn{2}{|c|}{$\begin{array}{c}\text { N1 = VERSIÓN } 1 \\
\text { N2 = VERSIÓN } 2 \\
\text { Colombia }\end{array}$}} & \multicolumn{2}{|c|}{$\begin{array}{l}\text { Prueba de } \\
\text { Levene para la } \\
\text { igualdad } \\
\text { de varianzas }\end{array}$} & \multicolumn{7}{|c|}{ Prueba t para la igualdad de medias } \\
\hline & & \multirow[b]{2}{*}{$F$} & \multirow[b]{2}{*}{$\begin{array}{l}P \\
\text { valor } \\
\text { Sig. }\end{array}$} & \multirow[b]{2}{*}{$\mathbf{t}$} & \multirow[b]{2}{*}{ gl } & \multirow[b]{2}{*}{$\begin{array}{l}\text { P valor } \\
\text { Sig.(bil } \\
\text { ateral) }\end{array}$} & \multirow[b]{2}{*}{$\begin{array}{l}\text { Dife- } \\
\text { rencia } \\
\text { de } \\
\text { medias }\end{array}$} & \multirow[b]{2}{*}{$\begin{array}{l}\text { Error } \\
\text { típ. de } \\
\text { la dife- } \\
\text { rencia }\end{array}$} & \multicolumn{2}{|c|}{$\begin{array}{l}95 \% \text { Intervalo de } \\
\text { confianza para la } \\
\text { diferencia }\end{array}$} \\
\hline & & & & & & & & & Inferior & Superior \\
\hline \multirow[t]{2}{*}{$\begin{array}{l}\text { Ejercicio } \\
3\end{array}$} & $\begin{array}{l}\text { Se han asu- } \\
\text { mido varian- } \\
\text { zas iguales }\end{array}$ & 1,172 & ,281 & $-1,350$ & 131 & , 179 &,- 118 & ,087 &,- 290 & ,055 \\
\hline & $\begin{array}{l}\text { No se han } \\
\text { asumido va- } \\
\text { rianzas } \\
\text { iguales }\end{array}$ & & & $-1,351$ & 126,539 &, 179 &,- 118 & 087 &,- 290 & 055 \\
\hline España & $F$ & $\begin{array}{l}\text { P } \\
\text { valor } \\
\text { Sig. }\end{array}$ & $\mathbf{t}$ & gl & $\begin{array}{l}\text { P valor } \\
\text { Sig.(bila } \\
\text { teral) }\end{array}$ & $\begin{array}{l}\text { Dife- } \\
\text { rencia } \\
\text { de } \\
\text { medias }\end{array}$ & $\begin{array}{l}\text { Error } \\
\text { típ. de } \\
\text { la } \\
\text { diferen- } \\
\text { cia }\end{array}$ & $\begin{array}{l}95 \% \text { In- } \\
\text { tervalo } \\
\text { de con- } \\
\text { fianza } \\
\text { para la } \\
\text { dife- } \\
\text { rencia }\end{array}$ & Inferior & Superior \\
\hline \multirow[t]{2}{*}{$\begin{array}{c}\text { Ejercicio } \\
1\end{array}$} & $\begin{array}{l}\text { Se han asu- } \\
\text { mido varian- } \\
\text { zas iguales }\end{array}$ & 3,509 & ,065 & $-1,975$ & 69 & ,052 & $-10,489$ & 5,312 & $-21,085$ & 108 \\
\hline & $\begin{array}{l}\text { No se han } \\
\text { asumido va- } \\
\text { rianzasiguales }\end{array}$ & & & $-1,980$ & 67,035 & ,052 & $-10,489$ & 5,297 & $-21,061$ & 083 \\
\hline \multirow[t]{2}{*}{$\begin{array}{c}\text { Ejercicio } \\
2\end{array}$} & $\begin{array}{l}\text { Se han asu- } \\
\text { mido varian- } \\
\text { zas iguales }\end{array}$ & 17,406 &, 000 & $-2,247$ & 69 & ,028 &,- 244 & 109 &,- 461 &,- 027 \\
\hline & $\begin{array}{l}\text { No se han } \\
\text { asumido va- } \\
\text { rianzas igua- } \\
\text { les }\end{array}$ & & & $-2,254$ & 66,711 & ,027 &,- 244 & , 108 &,- 461 &,- 028 \\
\hline \multirow[t]{2}{*}{$\begin{array}{c}\text { Ejercicio } \\
3\end{array}$} & $\begin{array}{l}\text { Se han asu- } \\
\text { mido varian- } \\
\text { zas iguales }\end{array}$ & 2,753 & ,102 & $-1,298$ & 69 & , 199 &,- 153 & ,118 &,- 389 & ,082 \\
\hline & $\begin{array}{l}\text { No se han } \\
\text { asumido va- } \\
\text { rianzas igua- } \\
\text { les }\end{array}$ & & & -1.297 & 68,676 & , 199 &,- 153 &, 118 &,- 389 & ,082 \\
\hline
\end{tabular}

Fuente: Elaboración propia. 
Heurísticos y sesgos cognitivos en la dirección de empresas: un meta-análisis Urra Urbieta, José Anastasio; Medina Lorza, Aida y Acosta Naranjo, Alejandro

Tabla 3

Porcentaje de confianza y frecuencia de F's identificadas en el Ejercicio 4

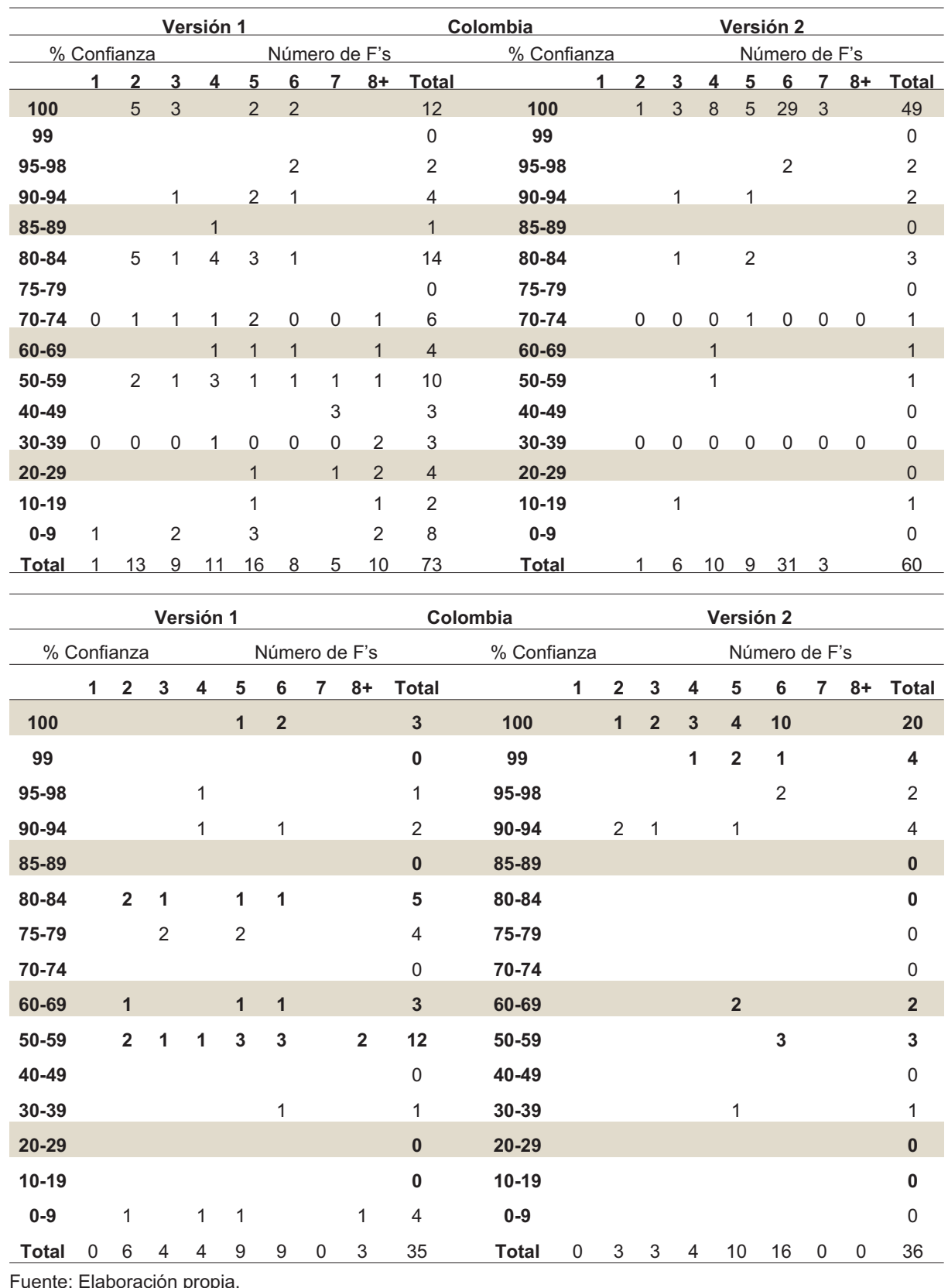


Luego, efectivamente, los heurísticos y sesgos cognitivos son propios de la naturaleza humana, e independientes de la cultura país, son interculturales. Adicionalmente, podemos afirmar que los heurísticos y sesgos cognitivos son resistentes al conocimiento, ya que como se puede apreciar en los resultados, aún en niveles altos de formación, las personas incurrimos en errores sin apercibirnos y convencidos de haber acertado en la diana.

\section{Conclusiones}

Resulta evidente que las personas somos parcialmente racionales, decidiendo y actuando en muchos de los casos bajo los efectos de los heurísticos cognitivos. Es decir, incurriendo en procesos normativos simplificadores de selección, procesamiento y ajuste de la información, que conducen a sesgos de valoración y predicción.

La naturaleza de los trabajos adscritos al campo de estudio del papel de los heurísticos en la Dirección de Empresas y en la estrategia es, en general, predominantemente teórica. Sin embargo, en las dos últimas décadas constatamos un incremento de las aportaciones empíricas sobre el papel de los heurísticos y sesgos en la estrategia empresarial, sobre todo en los procesos de adopción de decisiones estratégicas de emprendimiento, de alianzas, de adquisiciones; y ya en los últimos años, con predominio en campos como las finanzas y las decisiones de carácter financiero, la política, particularmente internacional, y el riesgo y su evaluación.
En cuanto a la evidencia empírica aportada por esta corriente de trabajos, podemos concluir que los de campo constituyen la excepción más que la regla. En este sentido, la mayor parte de la evidencia empírica se deriva de experimentos de laboratorio o de investigaciones con un diseño marcadamente experimental.

Aunque algunos trabajos señalan que los heurísticos y sesgos cognitivos podrían tener efectos beneficiosos durante formulación y/o la implementación de las decisiones estratégicas, la idea común y recurrente que las conclusiones tanto teóricas como empíricas sugieren es la del carácter pernicioso de los efectos derivados de los heurísticos y de los sesgos cognitivos. Sin embargo, las mismas aportaciones admiten que, si bien los heurísticos son no suprimibles por su naturaleza intrínsecamente humana, por su carácter de procesos podrían ser incididos y gestionados. En este sentido, algunos estudios apuntan que durante el proceso de adopción de decisiones directivas existen ciertos factores de contingencia, susceptibles de gestión, por tanto, la estructura del grupo decisor, la dinámica del grupo decisor y los elementos institucionales. Son factores, configurables $y$, por tanto, controlables por la dirección, presentando efectos directos sobre el mayor o menor desarrollo de ciertos heurísticos cognitivos durante el proceso de adopción de decisiones.

Finalmente, como demostramos empíricamente, los heurísticos y sesgos cognitivos existen, son interculturales y son resistentes al conocimiento. El mejor antídoto contra sus efectos perniciosos 
Heurísticos y sesgos cognitivos en la dirección de empresas: un meta-análisis Urra Urbieta, José Anastasio; Medina Lorza, Aida y Acosta Naranjo, Alejandro

en los procesos organizativos de adopción de decisiones es conocerlos, comprenderlos y ser conscientes de cómo actúan y de sus consecuencias; además de gestionar los factores de contingencia que inciden en el desarrollo de los mismos. Mostrarlos mediante ejercicios sencillos y exponer los resultados de su intervención se convierte en la mejor estrategia didáctica, si no la única, que facilita su aprehensión, su comprensión y su interiorización.

\section{Referencias bibliográficas}

Amason, Allen C. (1996). "Distinguishing the Effects of Functional and Dysfunctional Conflict on Strategic Decision Making: Resolving a Paradox for Top Management Teams", Academy of Management Journal, Vol. 39, $\mathrm{N}^{\circ} 1$, 123-148.

Amit, Raphael \& Schoemaker, Paul J.H. (1993). "Strategic Assets and Organizational Rent", Strategic Management Journal, Vol. 14, 33-46.

Bryant, Peter (2007). Self-regulation and decision heuristics in entrepreneurial opportunity evaluation and exploitation, Management Decision, Vol. 45, No. $4,732-748$.

Bukszar, Ed (1999). "Strategic Bias: The Impact of Cognitive Biases on Strategy", Canadian Journal of Administrative Sciences, Vol. 16, № 2, 105-117.

Busenitz, Lowell W. (1999). "Entrepreneurial Risk and Strategic Decision Making. It's a Matter of Perspective", Journal of Applied Behavioral Science, Vol. 35, № 3, September, 325-340.

Busenitz, Lowell W. \& Barney, Jay B. (1997). "Differences between Entrepreneurs and Managers in Large Organizations: Biases and Heuristics in Strate- gic Decision-Making", Journal of Business Venturing, Vol. 12, № 1, 9-30.

Child, John (1997). "Strategic Choice in the Analysis of Action, Structure, Organizations and Environment: Retrospect and Prospect", Organization Studies, Vol. 18, № 1, 43-76.

Cyert, Richard M. \& March, James G. (1963). A Behavioural Theory of the Firm, Prentice Hall, Englewood Cliffs.

Dean, James W. \& Sharfman, Mark P. (1993). "Procedural Rationality in the Strategic Decision-Making Process", Journal of Management Studies, Vol. 30, № 4, 587-610.

DiMaggio, Paul J. \& Powell, Walter W. (1983). "The iron cage revisited: institutional isomorphism and collective rationality in organizational fields", American Sociological Review, Vol. 48, 147160

Eisenhardt, Kathleen M. (1999). "Strategy as Strategic Decision Making", Sloan Management Review, Spring, 65-72.

Eisenhardt, Kathleen M. \& Zbaracki, Mark J. (1992). "Strategic Decision Making", Strategic Management Journal, Vol. 13, 17-37.

Fernández, Esteban (2009). Toma de decisiones: la previsible irracionalidad, en Martina Menguzzato, (Ed.) (2009). La Dirección de Empresas ante los retos del Siglo XXI. Homenaje al profesor Juan José Renau Piqueras, PUV, 113-123.

Fredrickson, James W. \& Laquinto, Anthony L. (1989). "Inertia and Creeping Rationality in Strategic Decision Processes", Academy of Management Journal, Vol. 32, No 3, 516-542.

Galbraith, Jay R. (1973). Designing Complex Organizations, Addison-Wesley, Massachusetts.

Gigerenzer, Gerd (1991). How to make cognitive illusions disappear: beyond "heuristics and biases", European Review 
of Social Psychology, Vol. 2, 83115.

Gigerenzer, Gerd (1993). The bounded rationality of probabilistic mental models, in K. I. Manktelow y D. E. Over (Eds.) (1993). Rationality: Psychological and philosophical perspectives, Routledge, London, 284-313.

Hambrick, Donald C. \& Mason, Phyllis A. (1984). "Upper Echelons: The Organization as a Reflection of Its Top Managers", Academy of Management Review, Vol. 9, № 2, 193-206.

Hannan, Michael T. \& Freeman, John H. (1977). "The Population Ecology of Organizations", American Journal of Sociology, Vol. 82, 929-964.

Jones, Paul E. \& Roelofsma, Peter H. M. P. (2000). "The potential for social contextual and group biases in team decisionmaking: biases, conditions and psychological mechanisms", Ergonomics, Vol. 43, Issue 8, August, 1129-1152.

Kahneman, Daniel \& Lovallo, Dan (1993). "Timid Choices and Bold Forecasts: A Cognitive Perspective on Risk Taking", Management Science, Vol. 39, № 1, January, 17- 31.

Krueger, Norris \& Dickson, Peter R. (1994). "How Believing in Ourselves Increases Risk Taking: Perceived Self-Efficacy and Opportunity Recognition", Decision Sciences, Vol. 23, $\mathrm{N}^{\circ} 3$, 385-400.

Langer, Ellen J. (1975)."The illusion of Control", Journal of Personality and Social Psychology, Vol. 32, N², 311328.

Langer, Ellen J. (1994). "The Illusion of Calculated Decisions", in Roger C. Schank y Ellen J. Langer (Eds.) (1994): Beliefs, Reasoning, and Decision Making. Psycho-Logic in Honor of Bob Abelson, Lawrence Erlbaum Associates, New Jersey, 33-53.
Lant, Theresa K.; Milliken, Frances J. \& Batra, Bipin (1992). "The Role of Managerial Learning and Interpretation in Strategic Persistence and Reorientation: An Empirical Exploration", Strategic Management Journal, Vol. 13, 585-608.

Lippmann, Walter (1922). Public opinion, Macmillan, New York.

Makridakis, Spyros G. (1990). Forecasting, Planning and Strategy for the 21st Century, Free Press, New York.

Menguzzato, Martina \& Urra, José Anastasio (2004). "Heurísticos y sesgos cognitivos en la decisión directiva de formación de alianzas estratégicas", Revue Sciences de Gestion, 41, 67-99.

Miller, C. Chet; Burke, Linda M. \& Glick, William H. (1998). "Cognitive Diversity among Upper-Echelon Executives: Implications for Strategic Decision Processes", Strategic Management Journal, Vol. 19, 39-58.

Miller, Kent D. \& Shapira, Zur (2003). "An empirical test of heuristics and biases affecting real option valuation", Strategic Management Journal, Vol. 25, $\mathrm{N}^{\circ} 3,269-284$.

Mintzberg, Henry (1998). "Crafting Strategy", in Henry Mintzberg, James Brian Quinn \& Sumantra Ghoshal (Eds.) (1998). The Strategy Process, Revised European Edition, Prentice Hall, Hemel Hempstead, 110-120.

Mintzberg, Henry; Ahlstrand, Bruce \& Lampel, Joseph (1998). Strategy Safari - The complete guide through the wilds of strategic management, Second Edition, Prentice Hall, London.

Murrell, Audrey J.; Stewart, Alice C. \& Engel, Brent T. (1993). "Consensus Versus Devil's Advocacy: The Influence of Decision Process and Task Structure on Strategic Decision Making", Journal of Business Communication, Vol. 30, No 4, 399-414. 
Heurísticos y sesgos cognitivos en la dirección de empresas: un meta-análisis

Urra Urbieta, José Anastasio; Medina Lorza, Aida y Acosta Naranjo, Alejandro

Nisbett, Richard E. \& Ross, Lee (1980). Human Inference: Strategies and Shortcomings of Social Judgment, Prentice-Hall, New Jersey.

Oliver, Christine (1991). "Strategic Responses to Institutional Processes", Academy of Management Review, Vol. 16, $\mathrm{N}^{\circ}$ 1: $145-179$.

Oliver, Christine (1997). "Sustainable Competitive Advantage: Combining Institutional and Resource-Based Views", Strategic Management Journal, Vol. 18, No 9, 697-713.

Palich, Leslie E. \& Bagby, D. Ray (1997). Using Cognitive Theory to Explain Entrepreneurial Risk-taking: Challenging Conventional Wisdom, Journal of Business Venturing, Vol. 10, № 6, 425438.

Pfeffer, Jeffrey \& Salancik, Gerald R. (1978). The External Control of Organizations: A Resource Dependence Perspective, Harper \& Row, New York.

Ross, Jerry \& Staw, Barry M. (1993). Organizational escalation and exit: Lessons from the Shoreham nuclear power plant, Academy of Management Journal, Vol. 36, № 4, August, 701732.

Russo, J. Edward \& Schoemaker, Paul J. H. (1992). Managing Overconfidence, Sloan Management Review, Winter, 7-17.

Schneider, Susan C. \& Angelmar, Reinhard (1993). Cognition in Organizational Analysis: Who's Minding the Store?, Organization Studies, Vol. $14, \mathrm{~N}^{\circ} 3$, 347-374.

Schoemaker, Paul J. H. (1990). Strategy, Complexity and Economic Rent, Management Science, Vol. 36, № 10, October, 1178-1192.

Schwenk, Charles R. (1984). Cognitive Simplification Processes in Strategic Deci- sion making, Strategic Management Journal, Vol. 5, 111-128.

Schwenk, Charles R. (1988). The Cognitive Perspective on Strategic Decision Making, Journal of Management Studies, Vol. 25, № 1, 41-55.

Scott, W. Richard (1987). The adolescence of institutional theory, Administrative Science Quarterly, Vol. 32, 493-511.

Shaver, Kelly G. \& Scott, Linda R. (1991). Person, Process, Choice: The Psychology of New Venture Creation, Entrepreneurship Theory and Practice, Vol. 16, № 2, Winter, 23-45.

Simon, Herbert Alexander (1957). Administrative Behaviour (2nd Ed.), MacmiIlan, New York.

Simon, Herbert Alexander (1978). Rationality as process and as product of thought, The American Economic Review, Vol. 68, № 2, 1-16.

Simon, Herbert Alexander (1983). Reason and Human Affairs, Stanford University Press, Stanford.

Simon, Mark; Houghton, Susan M. \& Aquino, Karl (1999). Cognitive Biases, Risk Perception, and Venture Formation: How Individuals Decide to Start Companies, Journal of Business Venturing, Vol. 15, № 2, 113-134.

Starbuck, William H. \& Milliken, Frances J. (1988). Executives Perceptual Filters: What They Notice and How They Make Sense, in Donald C. Hambrick (Ed.) (1988): The Executive Effect: Concepts and Methods for Studying Top Managers, JAI Press, Connecticut, 35-65.

Tversky, Amos N. \& Kahneman, Daniel (1974). "Judgment under Uncertainty: Heuristics and Biases", Science, Vol. 185, № 27, September, 1124-1131.

Tversky, Amos N. \& Kahneman, Daniel (1996). "On the reality of cognitive illusions", 
Psychological Review, Vol. 103, No 3, 582-591.

Urra, José Anastasio (2009). "La adopción de decisiones estratégicas en equipos directivos", en Martina Menguzzato (Ed.) (2009): La Dirección de Empresas ante los retos del Siglo XIX. Homenaje al profesor Juan José Renau Piqueras, PUV, 101-112.

Weick, Karl E. (1979). The Social Psychology of Organizing, Second Edition, Random House, New York.

Weick, Karl E.; Sutcliffe, Kathleen M. \& Obstfeld, David (2005). "Organizing and the Process of Sensemaking",
Organization Science, Vol. $16, \mathrm{~N}^{\circ} 4$, 409-421.

Wickham, Philip A. (2003). "The representativeness heuristic in judgments involving entrepreneurial success and failure", Management Decision, Vol. 41, No 2, 156-167.

Zapata, Gerardo y Canet, María Teresa (2009). "Individual Cognition: Reflections on its Processes and Influence in the Organization", Espacio Abierto, Vol. 18, N 2, 235-256.

Zucker, Lynne G. (1987). "Institutional theories of organization", Annual Review of Sociology, Vol. 13, 443-464. 